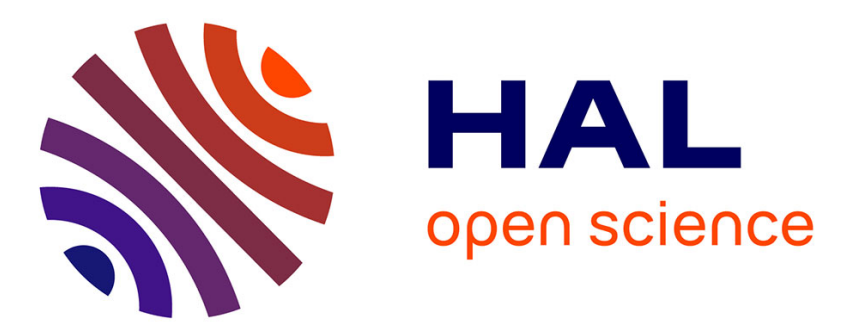

\title{
Development of a third-order accurate vorticity confinement scheme
}

Michel Costes, Ilias Petropoulos, Paola Cinnella

\section{To cite this version:}

Michel Costes, Ilias Petropoulos, Paola Cinnella. Development of a third-order accurate vorticity confinement scheme. Computers and Fluids, 2016, 136, pp.132-151. 10.1016/j.compfluid.2016.05.025 . hal-02877518

\section{HAL Id: hal-02877518 https://hal.science/hal-02877518}

Submitted on 22 Jun 2020

HAL is a multi-disciplinary open access archive for the deposit and dissemination of scientific research documents, whether they are published or not. The documents may come from teaching and research institutions in France or abroad, or from public or private research centers.
L'archive ouverte pluridisciplinaire HAL, est destinée au dépôt et à la diffusion de documents scientifiques de niveau recherche, publiés ou non, émanant des établissements d'enseignement et de recherche français ou étrangers, des laboratoires publics ou privés. 


\title{
Development of a third-order accurate vorticity confinement scheme
}

\author{
M. Costes ${ }^{\mathrm{a}, *}$, I. Petropoulos ${ }^{\mathrm{a}}$, P. Cinnella ${ }^{\mathrm{b}}$ \\ a ONERA, The French Aerospace Lab, 8 rue des Vertugadins, F-92190 Meudon, France \\ ${ }^{\mathrm{b}}$ DynFluid Lab., Arts et Métiers ParisTech, 151 Boulevard de l'Hopital, F-75013 Paris, France
}

\begin{abstract}
A B S T R A C T
A new 3rd-order Vorticity Confinement scheme is presented as an extension of the original VC2 scheme developed by Steinhoff for the resolution of the fluid dynamic equations. The theoretical developments are explained, and the method is tested. The results obtained show that the new scheme combines the accuracy of the underlying higher order scheme and the confinement capability of the original VC2 method.
\end{abstract}

Keywords:

Vorticity confinement

High-order schemes

Linear advection equation

Euler/RANS equations

Third-order VC2 confinement scheme

\section{Introduction}

The computation of vortices and wakes by CFD is a difficult problem. A large part of the methods applied in the past for wake simulations uses a Lagrangian approach [1,2], which allows a perfect conservation of the vortex sheets. However, most of them are restricted to inviscid, incompressible flows, and have difficulties to deal with the merging of vortical structures. The Eulerian approach is more general from all these aspects, but it suffers from other weaknesses. Numerical schemes for compressible flows need to be dissipative for stability reasons, thus wakes and vortices are diffused much faster in the computations than what actually occurs in reality. A significant amount of work has been done to reduce these flaws, considering either automatic mesh refinement in order to concentrate the mesh points in the vicinity of the vortical regions of interest, or the application of higher-order discretizations. Mesh adaption is generally performed with unstructured grids or with Cartesian grids and the Chimera overset grids method [36]. Grid adaption can be based on physical criteria or on error estimates, which may require the solution of an adjoint problem [7-10]. Higher-order space discretizations are an appealing alternative to decrease the dissipation of numerical schemes. However, the derivation of high-order space discretizations on general grids is complex and very often truly high accuracy is implemented on Cartesian grids only [11-13]. Whatever the approach, a significant

\footnotetext{
* Corresponding author. Fax: +33146734146.

E-mail addresses: mcostes@onera.fr (M. Costes), ilias.petropoulos@onera.fr (I. Petropoulos), paola.cinnella@ensam.eu (P. Cinnella).
}

additional CPU cost is needed, and the artificial spreading of wakes cannot be totally removed.

Alternative techniques in the Eulerian framework include the Vorticity Confinement method of Steinhoff [14-16], which proved to be very efficient for wake conservation. Such a method has been investigated at ONERA in the past [17-20]. We are more particularly interested here in the second VC scheme proposed by Steinhoff, known as VC2 [21,22]. In spite of its capability to maintain concentrated vorticity in the numerical simulations at a reduced extra cost with respect to fine mesh computations, the original VC formulation is only 1st-order accurate and the internal profile of the confined vortices is rapidly governed by the VC term. Therefore it is of little interest to use this method when a higher-order numerical scheme is applied, although the capabilities of VC could be beneficial even in this kind of simulation. This is more particularly the case when considering turbulent or separated flows for which vorticity plays a major role. The development of a VC method adapted to higher-order discretizations is therefore of interest. Furthermore, such a higher-order VC method may allow the level of negative dissipation applied in vortical regions to be decreased according to the lower numerical dissipation introduced by higher-order schemes.

In [23], higher-order confinement schemes were developed for the linear transport equation. The main outcome of this study is that all schemes asymptotically converge towards the same confined solution whatever the order of the confinement scheme, but the rate of convergence towards the asymptotic solution depends on the order of the scheme. The resulting schemes thus combine confinement property and high accuracy. The topic of the present paper is the extension of the 3rd-order VC scheme developed in 
[23] for the scalar linear advection equation to the Euler/RANS equations. A preliminary study including applications to helicopterrelated problems was presented in [24].

In the first part of this paper, the theoretical developments are presented, starting with a brief reminder of the basic 3rd-order confinement scheme for the linear 1D transport equation, and then describing its extension to the system of governing equations for gas dynamics. In a following part, the new approach is tested over simple test cases. First, a convergence study is performed for a steady 2D isentropic vortex in order to evaluate the actual accuracy of the various schemes used, with and without vorticity confinement. Then the advection of an inviscid 2D vortex over long distances is considered. The high-order VC scheme is compared with the original confinement scheme. Finally, a 2-D airfoil/vortex interaction is studied to investigate the capabilities of 3rd-order confinement alongside high-order schemes to capture the physics of a more realistic application.

\section{Presentation of the VC scheme}

\subsection{VC method for the $1 D$ linear transport equation}

The VC methodology was developed by Steinhoff et al. based on the theory of nonlinear solitary waves. Here we adopt a different approach, leading to similar results. Let us consider the simple case of the 1D transport equation:

$\frac{\partial u}{\partial t}+c \frac{\partial u}{\partial x}=0$

with $c>0$ the constant transport speed of a quantity $u \geq 0$. We start from a standard 1st-order upwind discretization on a Cartesian grid of constant space step $\Delta x$ and time step $\Delta t$ :

$u_{j}^{n+1}=u_{j}^{n}-\sigma \delta u_{j-1 / 2}^{n}$

where $u_{j}^{n}=u(j \Delta x, n \Delta t)$ is the numerical solution, $\delta u_{j-1 / 2}=u_{j}-$ $u_{j-1}$, and $\sigma=\frac{c \Delta t}{\Delta x}$ denotes the CFL number, with $\sigma<1$ for stability.

The original VC2 term uses a 2nd-difference of the harmonic mean between two successive grid values to correct the highlydiffusive 1st-order discretization of the above equation. Introducing the harmonic mean of the solution at two adjacent grid points, expressed as: $h\left(u_{j}, u_{j-1}\right)=\frac{2 u_{j} u_{j-1}}{u_{j}+u_{j-1}}$ for $u_{j} u_{j-1}>0$ and $h\left(u_{j}, u_{j-1}\right)=0$ otherwise, the 1st-order VC discretization of (1) writes:

$u_{j}^{n+1}=u_{j}^{n}-\sigma \delta u_{j-1 / 2}^{n}+\varepsilon \frac{\sigma(\sigma-1)}{2} \delta^{2} h\left(u_{j}^{n}, u_{j-1}^{n}\right)$

where $\delta^{2} h=\delta(\delta h)$ and $\varepsilon$ is a real constant called the confinement parameter. The flux difference correction due to the VC2 term is thus expressed by:

$\varepsilon \frac{1-\sigma}{2} \delta^{2} h\left(u_{j}^{n}, u_{j-1}^{n}\right)$

Deriving the equivalent partial differential equation from the linear differences of (3) and leaving the harmonic mean term unmodified leads to a mixed differential/difference equation representative of the numerical problem which is actually solved:

$$
\frac{\partial u}{\partial t}+c \frac{\partial u}{\partial x}-\frac{(1-\sigma) c \Delta x}{2}\left(\frac{\partial^{2} u}{\partial x^{2}}-\varepsilon \frac{\delta^{2} h\left(u, T_{-} u\right)}{\Delta x^{2}}\right)=0
$$

Here, $T_{-} u(x)=u(x-\Delta x)$ is the backward shifting operator so that $h\left(u, T_{-} u\right)$ is a symbolic representation of the harmonic mean between two successive node values of $u$. When $u \neq 0$, a Taylor expansion of the 2nd difference of the harmonic mean can be done, giving as leading term of the truncation error of (3):

$(\varepsilon-1) c \Delta x \frac{1-\sigma}{2} \frac{\partial^{2} u}{\partial x^{2}}$
Since $\sigma<1$, for $\varepsilon>1$ negative dissipation is introduced on the right hand side of (1) and the corresponding scheme, although 1st-order accurate, has the capability to conserve indefinitely nontrivial solutions which are transported at the correct speed by the numerical scheme. As shown in [23], these solutions have a pulse shape in the form $\operatorname{sech}(k(x-c t))$ and they are obtained by balancing the numerical diffusion of the first-order scheme and the nonlinear confinement term at the discrete level. As a result of eq. (5), asymptotic pulse solutions satisfy the equality:

$\frac{\partial^{2} u}{\partial x^{2}}-\varepsilon \frac{\delta^{2} h\left(u, T_{-} u\right)}{\Delta x^{2}}=0$

A sufficient condition for obtaining asymptotic solutions to the linear transport equation with confinement can be obtained by approximating $\frac{\partial^{2} u}{\partial x^{2}}$ on the same stencil as the harmonic mean difference in (7). Precisely, setting $\frac{\delta^{2} \mu u_{j-1 / 2}}{\Delta x^{2}}$, where $\mu u_{j-1 / 2}=$ $\frac{1}{2}\left(u_{j-1}+u_{j}\right)$ is the arithmetic averaging operator, this condition reduces to the simple relation between the arithmetic and the harmonic mean:

$\mu u_{j-1 / 2}=\varepsilon h\left(u_{j-1}, u_{j}\right)$

A nontrivial and nonsingular solution to (8) is given by:

$u_{j}=\operatorname{sech}\left(k x_{j}\right)=\frac{1}{\cosh \left(k x_{j}\right)}$

where $k$ is a positive real parameter such that $\varepsilon=\cosh \frac{k \Delta x}{2}$. As shown in [23], the counterpart of the numerical solution (9) is $u=\frac{1}{\cosh \left(a \frac{x}{\Delta x}\right)}$, where $a=k \Delta x$. Because $\varepsilon=\cosh \frac{a}{2}$ or, equivalently, $a=2 \ln \left(\varepsilon+\sqrt{\varepsilon^{2}-1}\right)$ it is clear that, for a prescribed $\varepsilon$, confined solutions depend on the mesh size because the signal is concentrated over the same number of cells, whatever the discretization. On the other hand, it is possible to compute the confinement parameter to keep a pulse solution close to the exact solution. In order to verify this assumption, it is possible to compute the numerical solution of (3) with the initial condition $u(x)=\frac{1}{\cosh (2.09 x)}$, corresponding to an asymptotic solution with $\varepsilon=1.6$ for $\Delta x=1$. The computational domain is a segment of length $L=100$ with periodicity boundary conditions at the left and right ends of the domain. The CFL number is set to $\sigma=0.611$, a typical value already used in [23] and [20]. The simulation is performed for various mesh refinements. Hereafter we consider $\Delta x=1, \Delta x=1 / 4$ and $\Delta x=1 / 16$. For the refined grids, the confinement parameter corresponding to $\varepsilon=\cosh \frac{a}{2}$ is $\varepsilon=1.03$ and $\varepsilon=1.002$ for $\Delta x=1 / 4$ and $\Delta x=1 / 16$ respectively. The solutions obtained after the signal has traveled a distance $c t=6110$ are compared with the exact one in Fig. 1, using the value of $\varepsilon$ corresponding to the mesh cell size of the simulation. The good preservation of the initial condition in the computation can be noted for all mesh cell sizes. Additionally, solutions for two other initial conditions corresponding to a Gaussian function with two values of the standard deviation, 2 and $1 / 2$, were computed using $\Delta x=1 / 2$ and $\varepsilon=1.14$. For the purpose of comparison, all initial conditions had the same integral versus $x$, equal to that of the hyperbolic secant initial condition. The results at time $c t=6110$ are compared with the solution using the hyperbolic secant as initial condition in Fig. 2. For readability, the results have been shifted by $x=5$ from one another, and the corresponding exact solutions as well. It is clear that whatever the initial condition, all pulse solutions converge towards the same asymptotic solution which is approximately described by the hyperbolic secant $u=\frac{1}{\cosh \left(a \frac{x}{\Delta x}\right)}$. This is confirmed by the time evolution of the discrete energy plotted in Fig. 3: all initial conditions converge towards a constant energy level, equal to that of the hyperbolic secant initial condition. 


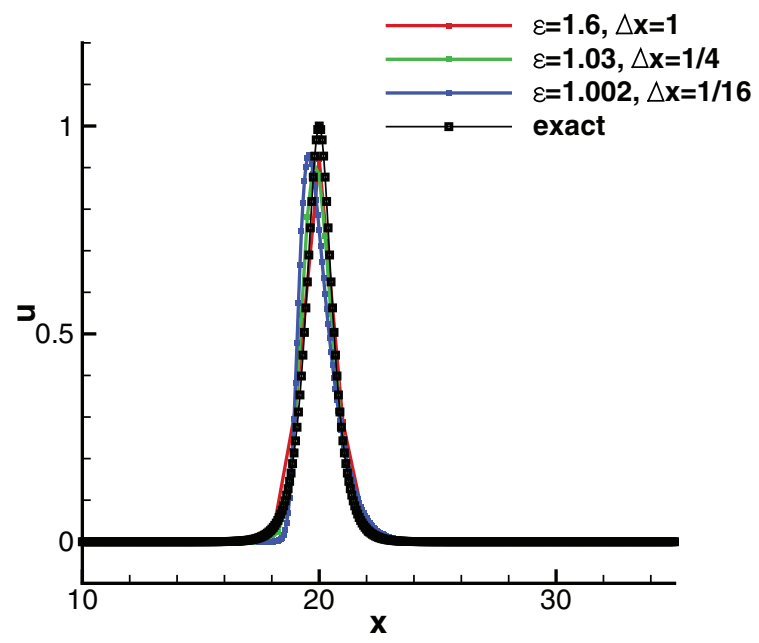

Fig. 1. Asymptotic solution at time $c t=6110$ with confinement parameter adapted to grid spacing.

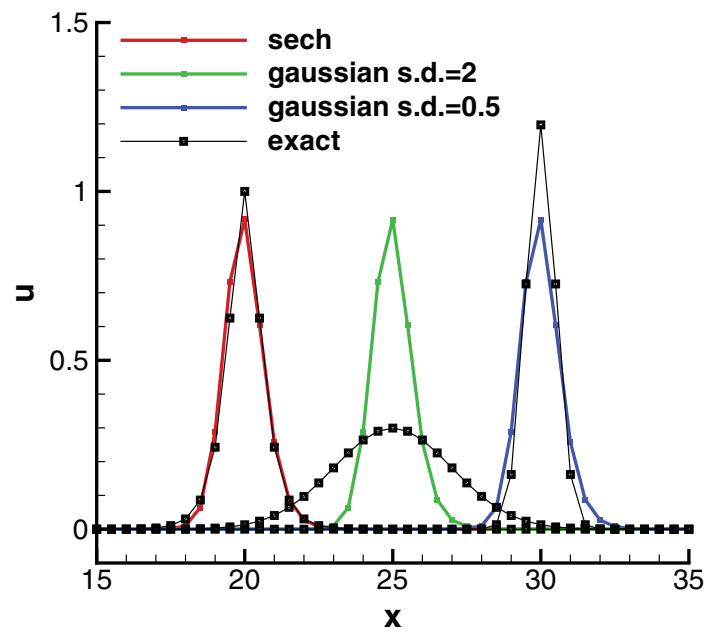

Fig. 2. Asymptotic solution at time $c t=6110$ for various initial conditions.

Finally, it is important to point out that if the VC2 term (4) is replaced by any linear second-difference approximation in Eq. (3), numerical stability requires $\varepsilon=1$. Since the leading term in the Taylor series development is identical between the nonlinear VC2 and any linear 2nd-difference, (6) shows that the linear schemes are at least 2nd-order accurate. According to the stencil used, the Lax-Wendroff, Warming-Beam and Fromm scheme can be obtained. Then the leading term in the truncation error has a 3rd derivative and is dispersive. Further, all these linear schemes are dissipative with a leading dissipative term given by the 4th derivative in their Taylor expansion. As a result, they asymptotically converge towards a constant solution for which the initial information is almost totally lost.

The VC2 scheme was extended to higher odd orders in [23], using the same ideas underlying the first-order approach. A nonlinear harmonic mean approximation of the opposite of the leading dissipative term of the truncation error of an odd-order linear scheme is added to the linear part of the scheme. At the 3rd-order of accuracy, the discretization becomes:

$$
\begin{aligned}
u_{j}^{n+1}= & u_{j}^{n}-\sigma \delta u_{j-1 / 2}^{n}+\frac{\sigma(\sigma-1)}{2 !} \delta^{2} u_{j}^{n}-\frac{(\sigma+1) \sigma(\sigma-1)}{3 !} \delta^{3} u_{j-1 / 2}^{n} \\
& +\varepsilon \frac{(\sigma+1) \sigma(\sigma-1)(\sigma-2)}{4 !} \delta^{4} h\left(u_{j}^{n}, u_{j-1}^{n}\right)
\end{aligned}
$$

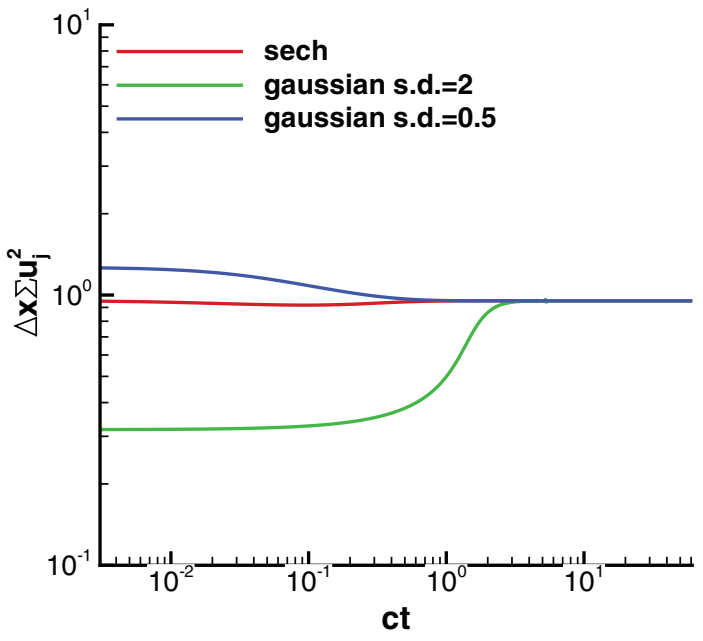

Fig. 3. Time evolution of energy norm for various initial conditions.

The 3rd-order VC flux difference correction is now:

$-\varepsilon \frac{(1+\sigma)(1-\sigma)(2-\sigma)}{4 !} \delta^{4} h\left(u_{j}^{n}, u_{j-1}^{n}\right)$

Accordingly, the leading term in the truncation error is:

$-(\varepsilon-1) c \Delta x^{3} \frac{(1+\sigma)(1-\sigma)(2-\sigma)}{4 !} \frac{\partial^{4} u}{\partial x^{4}}$

When $\varepsilon>1$, we have again negative dissipation added to the right hand side of (1), ensuring the confinement property of this 3rdorder accurate scheme. In [23], we showed that the VC schemes extended to any odd order $p$ are characterized by the same asymptotic pulse solutions which can be transported exactly at the discrete level. This is easily seen by deriving the equivalent equation similarly for the pth-order VC scheme:

$$
\frac{\partial u}{\partial t}+c \frac{\partial u}{\partial x}+(-1)^{\frac{p+1}{2}} O\left(\Delta x^{p}\right)\left(\frac{\partial^{p+1} u}{\partial x^{p+1}}-\varepsilon \frac{\delta^{p+1} h\left(u, T_{-} u\right)}{\Delta x^{p+1}}\right)=0
$$

Approximating the linear derivative with the same difference operator as the one used for the harmonic mean leads to the same sufficient condition as eq. (8), and thus to the same asymptotic solution. The interest of the higher-order schemes is that any initial solution converges to this asymptotic solution more slowly than the 1st-order VC, so that the benefits of higher-accuracy and of confinement can be combined. This is shown in the example below using the following 5th-order discretization with confinement:

$$
\begin{aligned}
u_{j}^{n+1}= & u_{j}^{n}-\sigma \delta u_{j-1 / 2}^{n}+\frac{\sigma(\sigma-1)}{2 !} \delta^{2} u_{j}^{n}-\frac{(\sigma+1) \cdots(\sigma-1)}{3 !} \delta^{3} u_{j-1 / 2}^{n} \\
& +\frac{(\sigma+1) \cdots(\sigma-2)}{4 !} \delta^{4} u_{j}^{n}-\frac{(\sigma+2) \cdots(\sigma-2)}{5 !} \delta^{5} u_{j-1 / 2}^{n} \\
& +\varepsilon \frac{(\sigma+2) \cdots(\sigma-3)}{6 !} \delta^{6} h\left(u_{j}^{n}, u_{j-1}^{n}\right)
\end{aligned}
$$

The 5th-order scheme was run for the same initial conditions considered before (hyperbolic secant, Gaussians with standard deviation of 2 and 1/2). As shown in Figs. 4 and 5, the numerical solutions corresponding to the Gaussian initial conditions evolve toward the hyperbolic secant asymptotic solution (and thus deviate from the exact Gaussian solution) after a much longer travelling distance $\left(c t>10^{4}\right)$ than the 1 st-order VC scheme, so that the original shape is still reasonably well conserved for $c t=6110$ for the less impulsive initial condition. This is also confirmed by inspection of the discrete energy of the solution presented in Fig. 6. The 


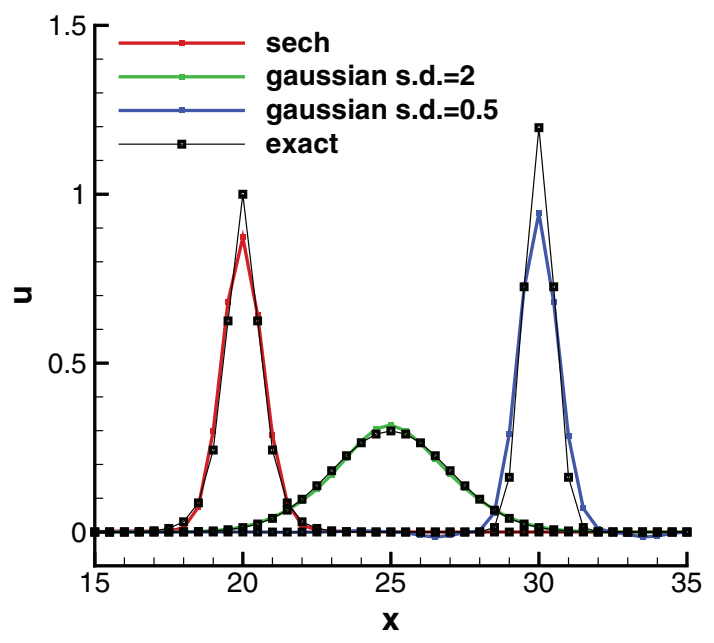

Fig. 4. Asymptotic solution at time $c t=6110$ for various initial conditions.

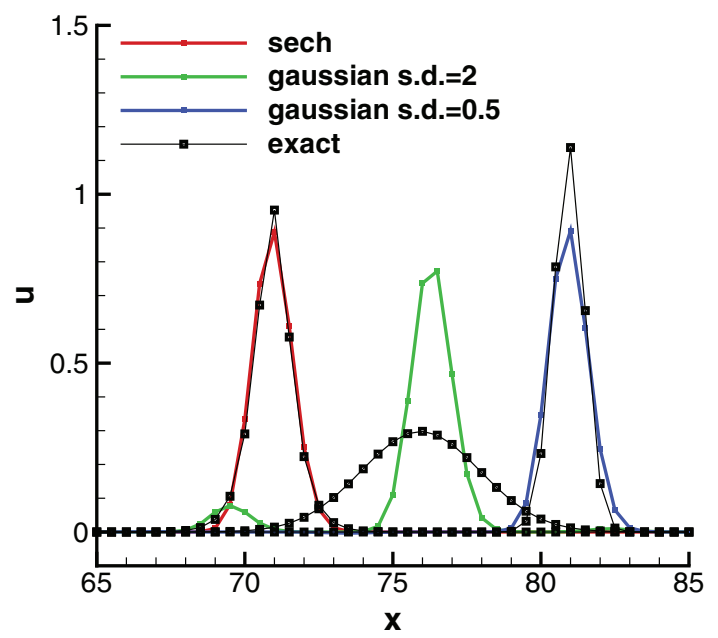

Fig. 5. Asymptotic solution at time $c t=4 \cdot 10^{8}$ for various initial conditions.

energy norm for the Gaussian initial condition with standard deviation of $1 / 2$ is much slower to converge to its asymptotic value. In this case, the 5th-order VC scheme maintains a small pulse at the foot of the initial one (Fig. 5). It comes from the known property of the VC scheme to split low frequency signals into higher frequency ones according to the value of $\varepsilon$ ([23]). It creates a small secondary pulse which is eventually slowly dissipated as the main pulse is being concentrated. This secondary pulse has to be completely removed in order to reach the asymptotic state.

\subsection{Extension to the Euler/RANS equations}

\subsubsection{First-order vorticity confinement}

Extending the VC2 schemes to the Euler/RANS equations is not straightforward because vorticity is not part of the conservative variables generally used in the resolution of these equations. In order to see how to translate the discretization from the linear transport equation to the fluid dynamic equations, let us come back to the original formulation of the VC2 scheme as described by Steinhoff. The VC term is included in the momentum equation as a source term. Denoting the primitive flow variables, density, velocity and pressure, by $\rho, \vec{U}$ and $p$, the viscous stress tensor by $\overline{\bar{\tau}}$ and the source term by $\vec{f}$, the momentum equation can be expressed

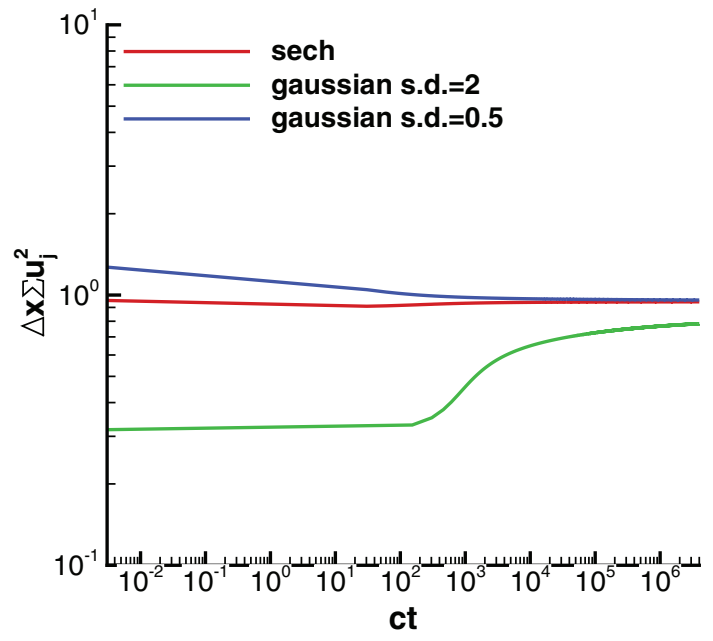

Fig. 6. Time evolution of energy norm for various initial conditions.

by:

$\frac{D \vec{U}}{D t}+\frac{1}{\rho} \vec{\nabla} p-\frac{1}{\rho} \vec{\nabla} \cdot \overline{\bar{\tau}}=\vec{f}$

with:

$\vec{f}=-\vec{\nabla} \times(\mu \vec{\omega}-\varepsilon \overrightarrow{\mathrm{w}})$

The first vector term on the right hand side of (16) is proportional to the vorticity vector $\vec{\omega}=\vec{\nabla} \times \vec{U}$, while the second one is aligned with vorticity, and its magnitude is equal to the harmonic mean of the vorticity modulus $\omega=\|\vec{\omega}\|$ of the surrounding grid points:

$\overrightarrow{\mathrm{w}}=\frac{\vec{\omega}}{\omega}\left[{\frac{\sum_{l=1}^{N} \omega_{l}^{-1}}{N}}^{-1}\right.$

\subsubsection{Analogy with linear transport equation}

The link between (16) and (4) can be established by deriving the vorticity transport equation, which is obtained by taking the curl of the momentum Eq. (15). We use a modified version of it for the specific vorticity in order to get rid of compressibility terms. This writes:

$\frac{d}{d t}\left(\frac{\vec{\omega}}{\rho}\right)=\overline{\bar{\nabla}} \vec{U} \cdot \frac{\vec{\omega}}{\rho}-\frac{1}{\rho} \vec{\nabla} \times \frac{\vec{\nabla} p}{\rho}+\frac{1}{\rho} \vec{\nabla} \times \frac{\vec{\nabla} \cdot \overline{\bar{\tau}}}{\rho}+\frac{1}{\rho} \vec{\nabla} \times \vec{f}$

Consider an isolated 2D vortex in inviscid flow. All terms except the last one on the right hand side of (18) vanish, because the velocity vector lies in a plane perpendicular to vorticity, and the gradients of pressure and density are aligned. Thus the VC term indirectly appears in the vorticity transport equation as:

$\left.\vec{\nabla} \times \vec{f}=\vec{\nabla}^{2}(\mu \vec{\omega}-\varepsilon \overrightarrow{\mathrm{W}})-\vec{\nabla} \vec{\nabla} \cdot(\mu \vec{\omega}-\varepsilon \overrightarrow{\mathrm{W}})\right)$

Again, the second term is equal to zero because:

$\vec{\nabla} \cdot \overrightarrow{\mathrm{w}}=\vec{\omega} \cdot \vec{\nabla} \frac{h(\omega)}{\omega}$

which is obviously zero for a $2 \mathrm{D}$ vortex. The vorticity transport equation can thus be written:

$\left.\left.\left.\frac{\partial}{\partial t} \frac{\omega}{\rho}\right)+\vec{U} \cdot \vec{\nabla} \frac{\omega}{\rho}\right)-\frac{\mu}{\rho} \vec{\nabla}^{2} \omega-\frac{\varepsilon}{\mu} h\left(\omega_{1}, \ldots, \omega_{N}\right)\right)=0$

The analogy of (21) with (5) is now clear. The first term of (16) is a diffusion term, which mimics the effect of the truncation error 
of the linear differences in (3). This contribution was found helpful for prescribing a linear dissipation term independently of the numerical scheme used. The second term in (16) is the anti-diffusive confinement term. The user-prescribed parameters, $\varepsilon$ and $\mu$, are both set proportional to the mesh size $\Delta x$ for consistency. Finally, the ratio $\frac{\varepsilon}{\mu}$ is the equivalent of the value of the confinement parameter $\varepsilon$ in the linear transport equation. By neglecting the dissipation of the baseline discretization, which is generally the case for $\mathrm{VC}$ of lower order than the underlying scheme, the numerical error of (21) can be attributed to the effect of the explicit dissipative VC term. In that case, the asymptotic solution of (21) is driven by the explicit term rather than the numerical error of the original equation. By analogy with the linear transport equation, an isolated vortex is expected to be convected without diffusion by the numerical scheme when:

$\omega_{i j}=\frac{\varepsilon}{\mu} h\left(\omega_{i+k j+l}\right)$

where $k$ and $l$ lie in the interval $[-1,1]$. Again, an asymptotic solution in the form $\omega_{i j}^{n}=\frac{1}{\cosh \left(K x_{i}+L y_{j}\right)}$ is recovered because:

$$
\begin{aligned}
& h\left(\omega_{i+k j+l}\right) \\
& \quad=\frac{9}{1+2 \cosh (K \Delta x)+2 \cosh (L \Delta y)+4 \cosh (K \Delta x) \cosh (L \Delta y)} \omega_{i j}
\end{aligned}
$$

and therefore:

$\frac{\varepsilon}{\mu}=\frac{1+2 \cosh (K \Delta x)+2 \cosh (L \Delta y)+4 \cosh (K \Delta x) \cosh (L \Delta y)}{9}$

$$
\geq 1
$$

Confinement is generally applied on Cartesian meshes with $\Delta x=$ $\Delta y$. Furthermore, the cross-section of the vortex has a symmetry of revolution, resulting in $K=L$. Similarly to the linear advection equation, introducing $a=K \Delta x=L \Delta y$, asymptotic solutions of VC2 can be written:

$\omega(x, y)=\frac{1}{\cosh \left(a\left(\frac{x}{\Delta x}+\frac{y}{\Delta y}\right)\right)}$

In that case:

$\frac{\varepsilon}{\mu}=\left(\frac{2 \cosh (a)+1}{3}\right)^{2}$

Therefore asymptotic solutions of VC2 depend directly on the mesh size for a given set of confinement parameters also for the Euler/RANS equations. Similarly to what was done with the linear advection, it is thus theoretically possible to adjust the value of $\varepsilon / \mu$ to a particular vortex size. This practice can be viable for some academic cases, but is not straightforward for complex flows and therefore has not been followed for the numerical results of the present study.

\subsubsection{Third-order vorticity confinement}

To derive a 3rd-order accurate VC2 confinement term $\vec{f}_{3}$, we need to introduce the equivalent of a 4th-difference operator (as in eq. (11)) in the vorticity transport equation. The idea is that, exactly as the 4th-difference in (11) can be obtained by recursively applying 1st-differences to the harmonic mean four times instead of two for the original VC2 term, applying the curl operator twice to the original VC term will naturally increase the order of the differencing by two since most of the terms disappear, leaving higherorder derivatives only. Introducing the vector $\vec{\alpha}=-(\mu \vec{\omega}-\varepsilon \overrightarrow{\mathrm{w}})$, the original VC2 term is given by:

$\vec{f}=\vec{\nabla} \times \vec{\alpha}$
Then, applying twice the curl operator to (27), we get:

$$
\begin{aligned}
\vec{\nabla} \times \vec{f} & =\vec{\nabla} \times(\vec{\nabla} \times \vec{\alpha})=\vec{\nabla}(\vec{\nabla} \cdot \vec{\alpha})-\vec{\nabla}^{2} \vec{\alpha} \\
\overrightarrow{f_{3}} & =\vec{\nabla} \times(\vec{\nabla} \times \vec{f})=\vec{\nabla} \times\left(\vec{\nabla}(\vec{\nabla} \cdot \vec{\alpha})-\vec{\nabla}^{2} \vec{\alpha}\right) \\
& =-\vec{\nabla} \times\left(\vec{\nabla}^{2} \vec{\alpha}\right)
\end{aligned}
$$

The 3rd order VC scheme is therefore obtained by taking the Laplacian of the original VC one. An interesting property of the Laplacian is that it is rotationally invariant, following the same ideas used by Steinhoff in the development of the VC method. Because our model equation is the vorticity transport equation, we are interested into the curl of the new confinement term, equal to:

$$
\begin{aligned}
\vec{\nabla} \times \overrightarrow{f_{3}} & =\vec{\nabla} \times(\vec{\nabla} \times(\vec{\nabla} \times \vec{f})) \\
& =-\vec{\nabla}\left(\vec{\nabla} \cdot\left(\vec{\nabla}^{2} \vec{\alpha}\right)\right)+\vec{\nabla}^{2}\left(\vec{\nabla}^{2} \vec{\alpha}\right)
\end{aligned}
$$

As for the original VC2 case, the first term on the right hand side of (29) vanishes for a 2D isolated vortex in inviscid flow since:

$$
\left.\vec{\nabla}^{2} \overrightarrow{\mathrm{W}}=\vec{\nabla} \vec{\nabla} \cdot \overrightarrow{\mathrm{W}}-\vec{\nabla} \times \vec{\nabla} \times \overrightarrow{\mathrm{W}}\right)
$$

The first term on the right hand side of (30) was found to be zero due to relation (20), while the second one obviously vanishes when applying the divergence operator to the first term on the right hand side of (29). Therefore the confinement term (29) is fully similar to (21), with the sum of a dissipation and a confinement term. The successive applications of the curl operator naturally introduce the alternate sign of the dissipative terms with the increasing order of the derivatives. Finally, using undivided differences in the computation of the Laplacian of the components of the original VC2 term will naturally provide the correct order of discretization:

$\overrightarrow{f_{3}}=\epsilon_{I J K} \frac{\delta_{I}}{\Delta x_{I}}\left(\delta_{1}^{2}+\delta_{2}^{2}+\delta_{3}^{2}\right)\left(\mu \omega_{J}-\varepsilon \mathrm{w}_{J}\right) \vec{e}_{K}$

where the directional difference operators are defined by:

$$
\begin{aligned}
& \left(\delta_{1} u\right)_{j+1 / 2, k, l}=u_{j+1, k, l}-u_{j, k, l} \\
& \left(\delta_{2} u\right)_{j, k+1 / 2, l}=u_{j, k+1, l}-u_{j, k, l} \\
& \left(\delta_{3} u\right)_{j, k, l+1 / 2}=u_{j, k, l+1}-u_{j, k, l}
\end{aligned}
$$

Here we have used index notations and Einstein summation convention, with $I, J, K$ varying from 1 to $3, \epsilon_{I J K}$ the Levi-Civita alternating symbol and $\overrightarrow{e_{1}}, \overrightarrow{e_{2}}, \overrightarrow{e_{3}}$ the unit vectors along the three directions of the Cartesian frame. Since the original VC2 scheme is 1 st-order accurate, the proposed VC term is now 3rd-order accurate.

\subsection{Numerical method}

The confinement schemes described above have been implemented in the Cassiopée Cartesian solver of the elsA suite [25], which solves the Euler and Navier-Stokes equations in a finitevolume approach. Various discretization schemes are available, including the centered 2nd-order space discretization with Jameson's artificial viscosity term, and the non-compact 3rd-order and 5thorder directional schemes described by Saunier et al. in [26] and derived by Lerat et al. in [27]. These schemes can be assimilated to 3rd-order and 5th-order MUSCL schemes using high-order extrapolation of the fluxes. Thanks to the equivalence between the finitevolume and finite-difference approach on Cartesian grids, their implementation can be greatly simplified with respect to curvilinear grids, writing in the case of the 2D Euler equations in semi-discrete 
form:

$$
\begin{aligned}
\frac{\partial W}{\partial t} & \left.+\frac{1}{\Delta x} \delta_{1} I-\frac{1}{6} \delta_{1}^{2}+\frac{1}{30} \delta_{1}^{4}\right) \mu_{1} F \\
& \left.+\frac{1}{\Delta y} \delta_{2} I-\frac{1}{6} \delta_{2}^{2}+\frac{1}{30} \delta_{2}^{4}\right) \mu_{2} G=0
\end{aligned}
$$

$W$ is the vector of conservative variables, $F$ and $G$ are the flux vectors along the $x$ and $y$ directions respectively, $\delta_{1}$ and $\delta_{2}$ are the difference operators defined in (32) and:

$$
\begin{aligned}
& \left(\mu_{1} F\right)_{j+1 / 2, k}=\frac{1}{2}\left(F_{j, k}+F_{j+1, k}\right) \\
& \left(\mu_{2} G\right)_{j, k+1 / 2}=\frac{1}{2}\left(G_{j, k}+G_{j, k+1}\right)
\end{aligned}
$$

are the classical averaging operators along the grid directions. The discretisation (33) is 6th-order accurate, non-dissipative. For stability, a 5th-order artificial viscosity term is added to the right hand side:

$\left(\frac{1}{\Delta x} \delta_{1}\left(\frac{\left|A_{R}\right|}{60} \delta_{1}^{5}\right)+\frac{1}{\Delta y} \delta_{2}\left(\frac{\left|B_{R}\right|}{60} \delta_{2}^{5}\right)\right) W$

where $A_{R}$ and $B_{R}$ are Roe linearizations of the Jacobian matrices $A=\frac{\partial F}{\partial W}$ and $B=\frac{\partial G}{\partial W}$. The 3rd-order scheme is similarly obtained by dropping the 4 th derivatives in (33) and modifying the artificial dissipation accordingly.

The vorticity confinement term (31) is added to the right hand side of the momentum equation, using standard 2nd-order finitedifferences. The VC method was implemented in a Cartesian solver only. The regions of the flowfield where the confinement term is applied are selected by the Q criterion: all parts of the flowfield for which the $\mathrm{Q}$ criterion is above a user-prescribed threshold value are selected as regions of concentrated vorticity to confine. To compute the flow around obstacles, curvilinear body grids are required and the Chimera overset grid method is used $[28,29]$. Computations with confinement thus require three user-prescribed input quantities: $\varepsilon, \mu$ and the threshold value for the $\mathrm{Q}$ criterion. In practice, the influence of this last parameter is quite small as it is only used for selecting regions of concentrated vorticity in the flowfield.

\section{Application of the method}

\subsection{Steady $2 D$ isentropic vortex}

As a first test case, we perform a grid convergence study for the steady isentropic 2D vortex already considered by Yee et al. in [30]. The objective is to check that the confinement terms follow the expected convergence rate according to the baseline scheme used. The vortex is set in the fluid at rest, and the Euler equations are solved for a fixed period of time. The final solution is then compared to the initial one in order to estimate the error introduced by the numerical scheme, assuming that the results should be identical. This would indeed be the case if the isentropic vortex was an exact solution to the Euler equation, but it is only approximate. Indeed, Crocco's form of Euler's momentum equation for steady-state homentropic flow reduces to:

$\vec{U} \times \vec{\omega}+T \vec{\nabla} s=0$

Since the vorticity and velocity vectors are perpendicular, the flow cannot remain fully isentropic, but the entropy gradient is small, thus justifying the analysis below.

The computed flow domain is square $[0,10] \times[-5,5]$, and the vortex is set in the middle at $x=5, y=0$. Six grids of increasing fineness have been considered, halving the mesh cell size from one mesh to the following one. The coarsest mesh has 441 points, corresponding to a mesh cell size $\Delta x=\Delta y=0.5$, and the finest one has 410,881 points and a mesh cell size of $\Delta x=\Delta y=0.015625$. All computations were run between time $t=0$ and $t=1$ with a time step $\Delta t=2 \cdot 10^{-4} \Delta x$ sufficiently small to introduce negligible time-discretization errors. The L2 norm of the difference between the initial and final solutions was then computed for the conservative variables and entropy. The L2 error for entropy is plotted in Fig. 7 for the 2nd, 3rd and 5th-order schemes alone (baseline versions) and with 1st-order and 3rd-order confinement. When confinement is applied, the confinement parameters are constant and set to $\varepsilon=0.16$ and $\frac{\varepsilon}{\mu}=1.67$. These values are based on a small parametric study in order to check that they allow a good preservation of vorticity for a sufficienly long period of time with the coarse grid. This grid convergence study was also repeated for other values of the confinement parameters and did not show any difference on the convergence rates, so that the plotted results are meaningful. The corresponding theoretical convergence orders of the schemes are also plotted in dashed lines for convenience. As expected, the nominal order of convergence of the baseline schemes is recovered by the numerical solutions as soon as the mesh is fine enough. For all cases, confinement reduces the order of convergence to the order of the confinement term (first or third order respectively), thus showing the interest of increasing the order of the confinement term when higher-order flux discretizations are used. Again, sufficiently fine meshes are necessary to get approximately the nominal order of convergence of the discretization.

\subsection{Advection of an inviscid $2 D$ vortex}

For assessing the capability of confinement to maintain concentrated vorticity in the numerical solution, we consider a test case similar to that computed by Yee et al. in [31], namely the advection of an isolated 2D vortex in an inviscid flow, but instead of using the isentropic vortex of the previous section, a vortex model described by Visbal et al. was used [32]. The exact solution is just the passive advection of the initial vortex at the freestream velocity if this initial vortex is an exact solution to the Euler equation. As this is not the case, the vortex will be modified and converge to a true solution of the Euler equations which is advected across the flow domain. Since the goal of this application is to investigate the vortex preservation capability of the numerical schemes, the difference between the initial and final structure of the vortex is of minor importance.

In the following, the capability of the baseline 2nd-, 3rd- and 5 th-order schemes for this problem is first assessed. The dissipative and dispersive properties of the schemes with and without confinement are investigated through their capability to capture the trajectory and the strength of the vortex during its advection. The error in trajectory is due to dispersion and the error in strength comes from the dissipation of the schemes. The minimum density at the vortex center is used to characterize the vortex strength and position. Then the effect of 1st- and 3rd-order confinement with these baseline schemes is investigated for constant values of the confinement parameters. These empirical values were determined again from a preliminary parametric study. A sensitivity study to confinement parameters is then performed and its effect on the vortex solutions is analyzed. Since the role of vorticity confinement is to contribute a negative dissipation in vortical regions, it is expected that both 1st- and 3rd-order confinement have little effect on vortex trajectory, but reduce vortex dissipation significantly. Furthermore, the initial structure of the vortex should be better preserved with 3rd-order confinement as convergence to the asymptotic state of the confinement term is slower. On the contrary, for very long advection times, both 1st- and 3rd-order confinement are expected to converge towards similar asymptotic 


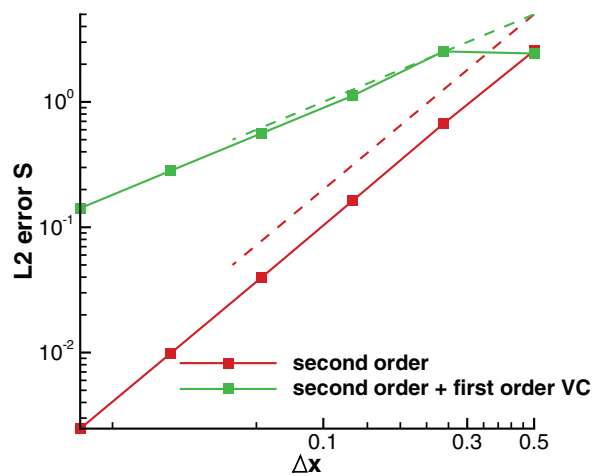

(a) 2nd-order scheme

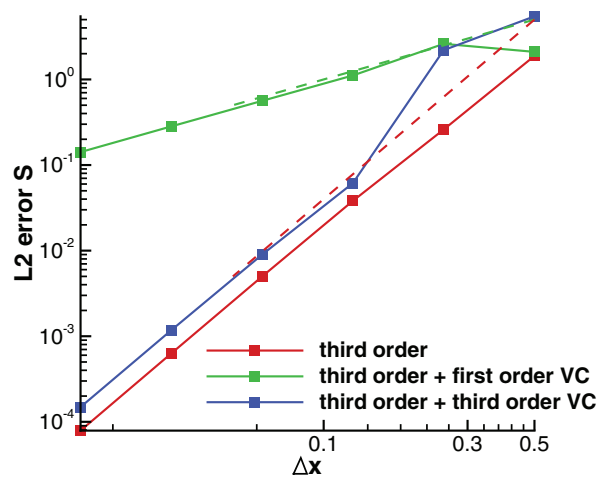

(b) 3rd-order scheme

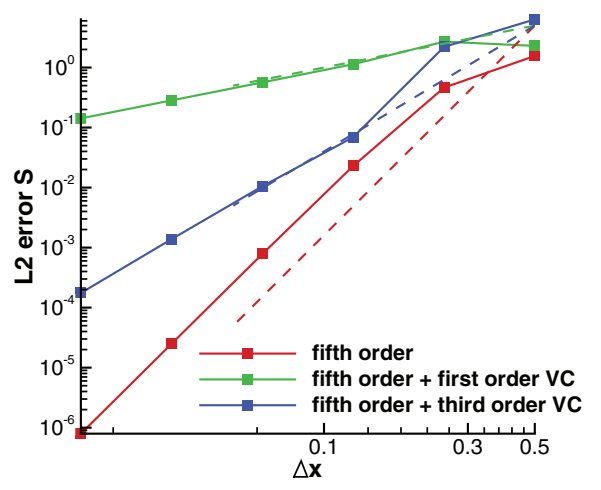

(c) 5th-order scheme

Fig. 7. L2 error for entropy with 2nd (a), 3rd (b) and 5th-order schemes (c) without and with confinement.

solutions which are transported across the grid without being modified.

The computational domain consists of a box $[0,110] \times[-5,5]$ with periodicity conditions applied along all four boundaries. Three grid levels are considered: fine, medium and coarse with a resolution corresponding to the three coarsest meshes of the preceding grid convergence study. The cell size of the Cartesian mesh is thus equal respectively to $\Delta x=\Delta y=0.125 / 0.25 / 0.5$ for the fine/medium/coarse grids. The initial vortex is set at $x=5, y=0$, and the number of grid points in the vortex core radius is roughly equal to $12 / 6 / 3$ for the fine/medium/coarse grids. The initial condition discretized on the three grids is presented in Fig. 8. Note that, because too large vortex diffusion without confinement led to an interaction of the vortex with the upper and lower periodicity conditions after a while, the upper and lower boundaries were set at $y= \pm 10$ for the coarse mesh computations.

The compressible Euler equations are solved using a freestream Mach number of 0.2 . The solution is explicitly advanced in time using a classical 4-step Runge-Kutta scheme, using a time step equivalent to $U_{\infty} \Delta t=0.02$, so that a distance $L=100$ is traveled in 25,000 time steps. Computations were run over 300,000 time steps, corresponding to a travel length of $L=1200$ at the freestream velocity (a distance roughly similar to that computed by Yee et al. in [31]). The density contours are plotted every 25,000 time steps so that, considering the length of the grid box and the periodicity conditions, the vortex is switched back by $\Delta X=10$ from plot to plot, starting at $X=105$ after 25,000 time steps, and the final solution after 300,000 time steps should be located again at $X=105$ and superimposed with the one after 25,000 time steps. An example of result is shown in Fig. 9 for the 2nd-, 3rd and 5th-order baseline schemes, clearly showing the effect of dissi- pation and dispersion on the solution. From 2nd- (Fig. 9(c)) to 3rd-order (Fig. 9(b)), the large reduction of dispersion error results in an improved vortex trajectory, while both schemes have similar dissipation properties resulting in a loss of the vortex after 5 to 6 passages. Switching to 5th-order (Fig. 9(a)) significantly reduces the dissipation errors, and the vortex is reasonably well captured all along the period of time considered. Note however that, even with the 5th-order scheme and the fine mesh vorticity is dissipated over time. Another measure of vortex dissipation is the time evolution of minimum density, plotted in Fig. 10 for the three grids. Density at the vortex centre tends to rise up to values close to the freestream density. The 5th-order scheme keeps a more concentrated vortex much longer than lower order ones, albeit the effect of numerical dissipation becomes evident after a sufficiently long time elapsed (Fig. 10(a)). When considering the medium (Fig. 10(c)) and the coarse mesh (Fig. 10(e)), the vortex is rapidly dissipated whatever the order of the numerical scheme.

The introduction of vorticity confinement changes the situation drastically. With the 2nd-order scheme only 1st-order confinement was used, while both 1 st-order and 3rd-order confinement were used with the 3rd-order and 5th-order schemes. For the 2nd- and 3rd-order schemes, the confinement parameters were set to $\varepsilon=0.16, \varepsilon / \mu=1.67$. For the 5th-order scheme, values of $\varepsilon=$ $0.02, \varepsilon / \mu=1.67$ were used except with the coarse mesh where we had to double $\varepsilon=0.04$ with 1 st-order confinement in order to maintain the vortex in the solution, the lower value $\varepsilon=0.02$ leading to a progressive dissipation of the vortex in the simulation. When considering fine mesh results (Fig. 10(b)), all solutions computed with confinement converge towards a more concentrated vortex profile than the original one with smaller value of density. A similar asymptotic state is reached whatever the order of 


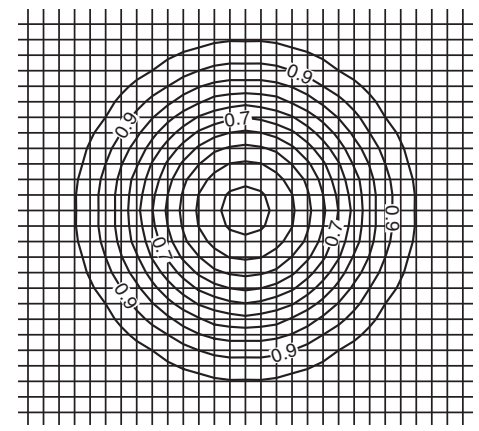

(a) Fine mesh

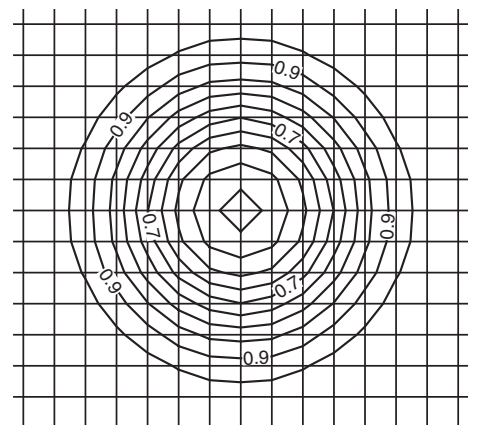

(b) Medium mesh

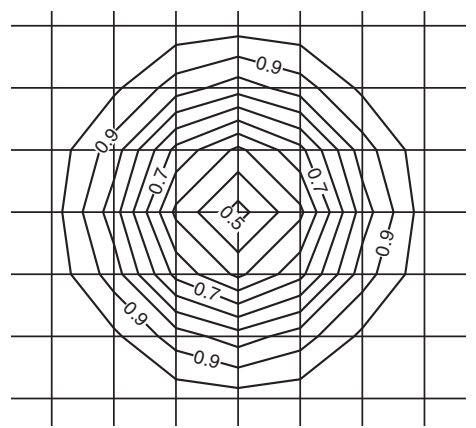

(c) Coarse mesh

Fig. 8. Iso-density contours of the initial vortex on the fine (a), medium (b) and coarse (c) meshes.

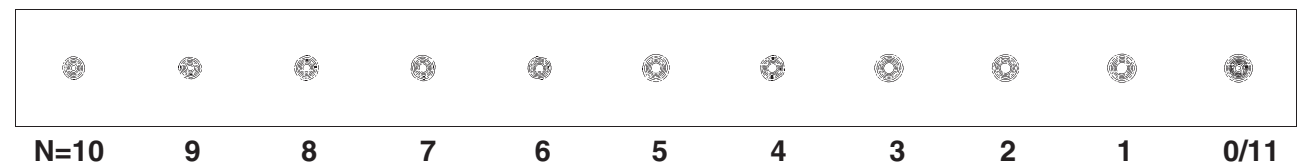

(a) 5th-order

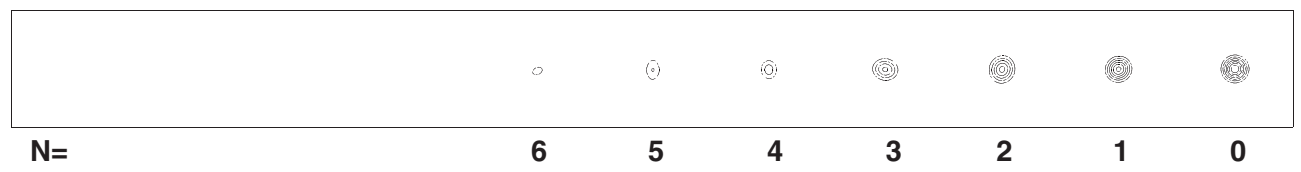

(b) 3rd-order

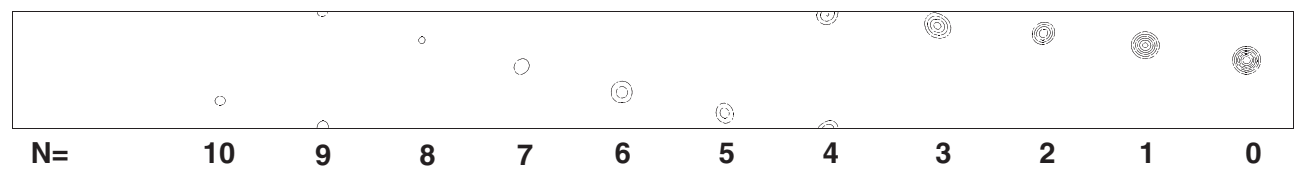

(c) 2nd-order

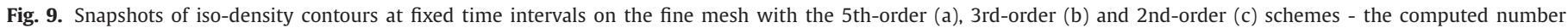
of passages $(\mathrm{N})$ across the domain is labelled below each vortex.

confinement and scheme. However, computations with 3rd-order confinement converge slower to their asymptotic state, more especially when the 5 th-order scheme is used. The situation with the medium mesh is different (Fig. 10(d)). With 1st-order confinement, all numerical schemes also converge towards a same asymptotic state, but at a slower rate than with the fine mesh. The resulting vortex is also more concentrated than the original one, but less than with the fine mesh. On the contrary, computations with 3rd-order confinement remain much closer to the initial vortex over the computed period of time. Nevertheless, the slow decrease of density at the vortex centre over time gives an indication that the vortex is asymptotically converging towards the same structure as with 1 st order confinement. Finally, for the coarse mesh (Fig. 10(f)), the vortex is maintained in the solution with all 


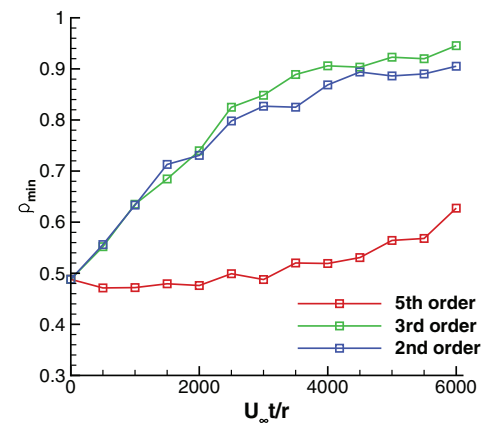

(a) fine

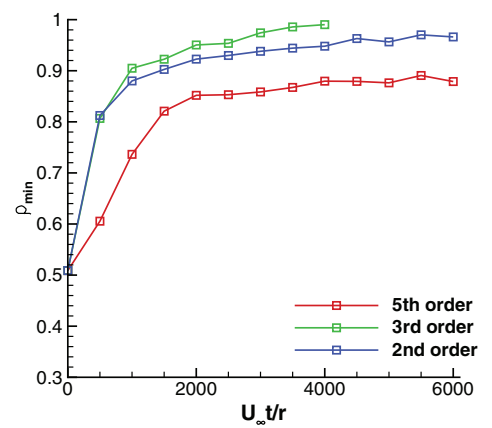

(c) medium

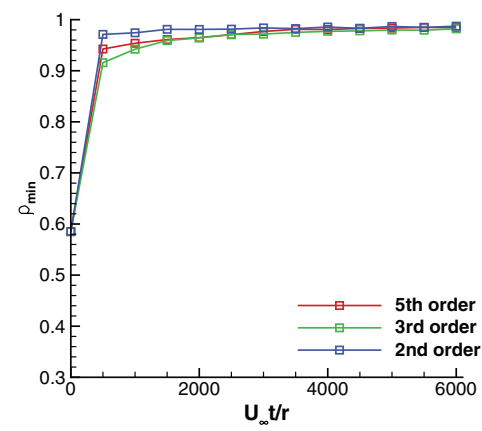

(e) coarse

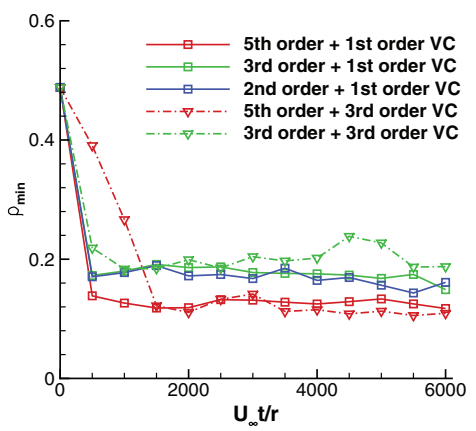

(b) fine, $\varepsilon=$ $0.02 / 0.16 / 0.16, \varepsilon / \mu=1.67$

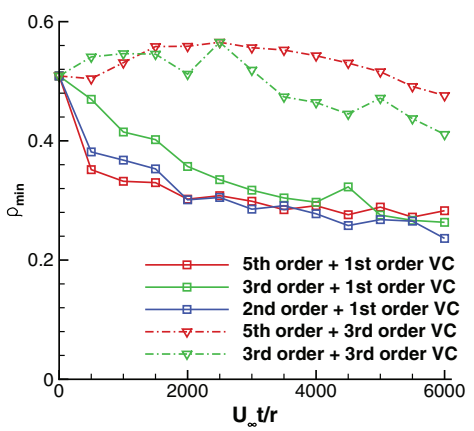

(d) med., $\varepsilon=$ $0.02 / 0.16 / 0.16, \varepsilon / \mu=1.67$

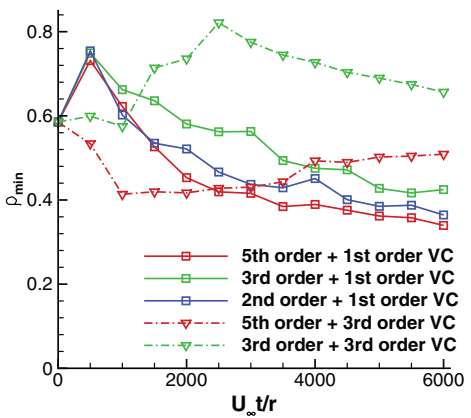

(f) coarse, $\varepsilon=$ $0.04 / 0.16 / 0.16, \varepsilon / \mu=1.67$

Fig. 10. Time evolution of the minimum density at the vortex centre on the fine (a-b), medium (c-d) and coarse (e-f) mesh.

confinement schemes. Again, all computations with 1st-order confinement seem to converge towards the same asymptotic state, but the asymptote is yet longer to reach than with the medium mesh. The computations with 3rd-order confinement have not yet reached their asymptotic state.

In order to better analyze the solutions, two examples of density profiles across the vortex are plotted after 25,000 time steps, i.e. after the vortex has crossed the computational domain. In Fig. 11, the effect of the confinement order is compared for the same 5th-order scheme. Generally, the 3rd-order confinement term provides solutions in closer agreement with the exact one during this "early" phase of the computation, whatever the mesh used. The fine and coarse mesh show a small overestimation of vortex strength while the agreement is almost perfect with the medium grid. On the contrary, with 1st-order confinement, the vortex strength is strongly overestimated with the fine mesh and underestimated with the coarse mesh, although the value of $\varepsilon$ was doubled in this last case. As a matter of fact, only the medium mesh provides a moderate overestimation of vortex strength at this time of the computation. The results obtained without confinement are also plotted, showing the benefits of confinement schemes, especially for the medium and the coarse mesh.

The density profiles obtained with the 2nd-order, 3rd-order and 5 th-order schemes with 1st-order confinement and 3rd-order confinement are compared in Fig. 12. Again, the better capability of 3rd-order confinement to maintain the initial vortex during this early phase of the advection can be noted. Furthermore, for the same 3rd-order confinement term, the superiority of the 5th-order 


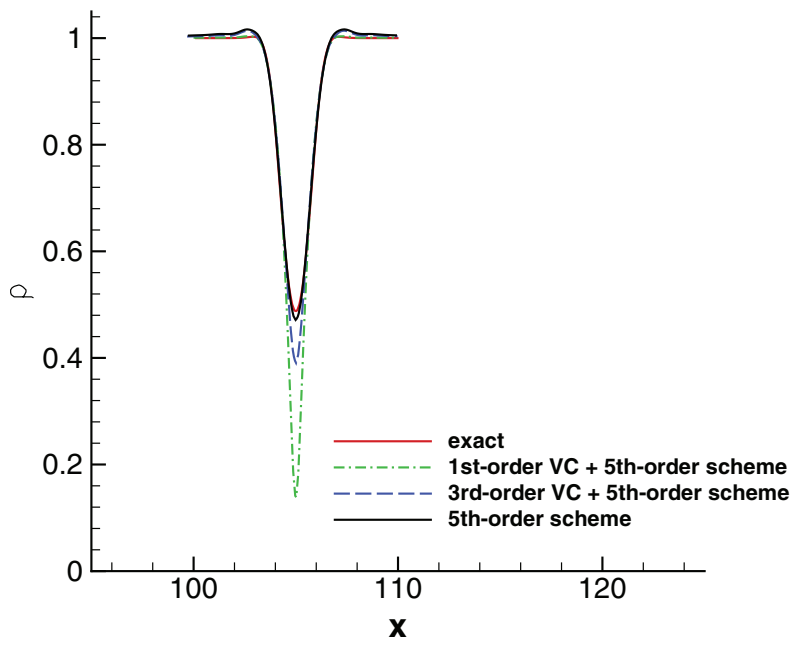

(a) fine, $\varepsilon=0.02, \varepsilon / \mu=1.67$

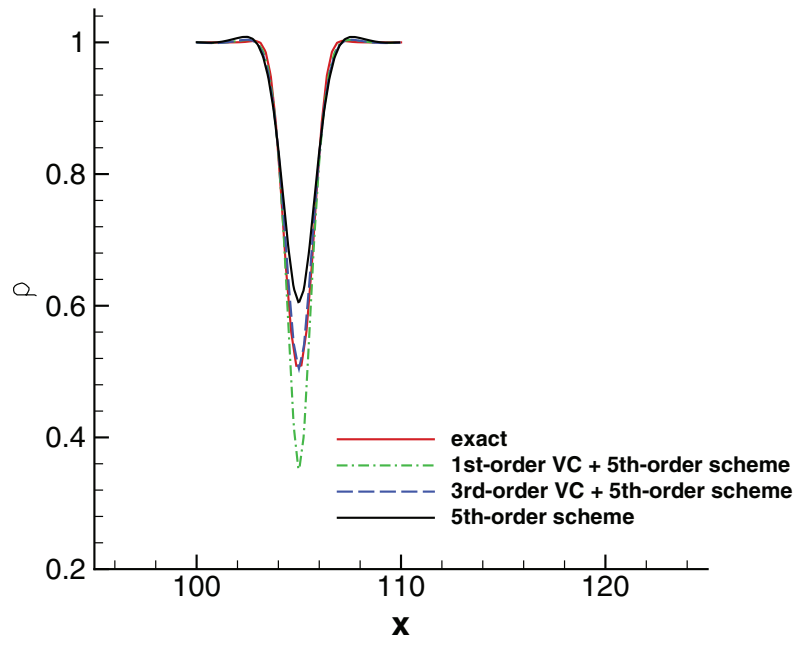

(b) medium, $\varepsilon=0.02, \varepsilon / \mu=1.67$

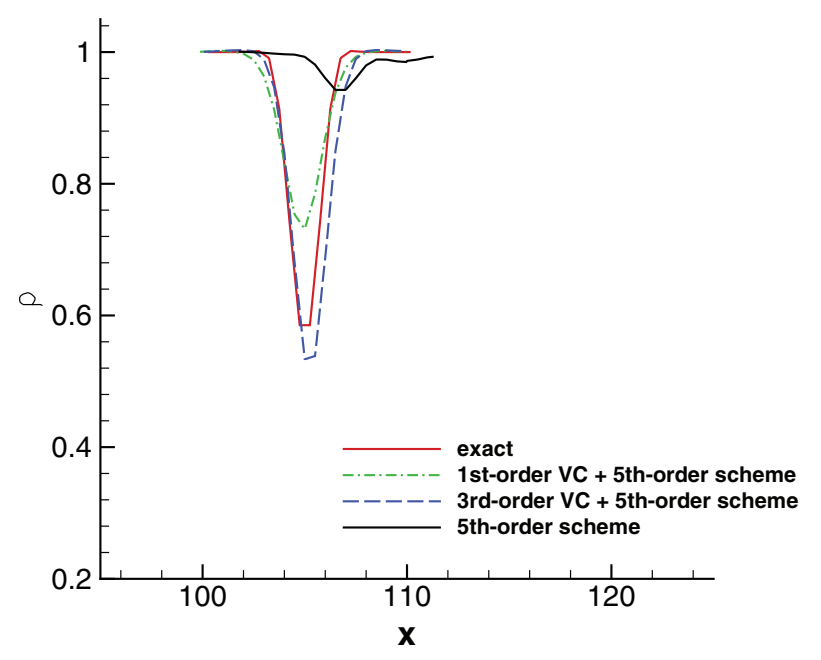

(c) coarse, $\varepsilon=0.04, \varepsilon / \mu=1.67$

Fig. 11. Density profiles across the vortex after 25,000 time steps with the 5th-order scheme for the fine (a), medium (b) and coarse (c) grids.

scheme over the 3rd-order scheme is also clear. The reason is that, because the numerical dissipation of the 5 th-order scheme is lower, the magnitude of the confinement parameters is also reduced by a factor of 4 to 8 with respect to the 3rd-order scheme. Finally, the lower dispersion error of the 5th-order scheme results in a more accurate prediction of vortex position at this time of the computation.

When very long periods of time are considered (300,000 time steps, Fig. 13), confinement has a stronger effect on the vortex density profile. As already noted before, computations with 1 st-order confinement are in their asymptotic range and the computed vortex is significantly stronger than the original one with a minimum density much smaller than the exact solution. The solution is also fairly similar for all of the three grids considered. However, the cumulated dispersion error over time is different from grid to grid with a phase lead for the fine and medium grid and an apparent phase lag for the coarse grid (as will be discussed below, the com- puted vortex position is actually ahead of the exact one of a little less than one mesh domain length). With 3rd-order confinement, the vortex position is generally more accurate, due to the reduced dispersion error of the underlying 3rd- and 5th-order scheme. For the medium and the coarse mesh, the density profiles are also close to the exact solution, while the fine mesh solution is close to that obtained with 1st-order confinement. In this last case, this is because both 1st-order and 3rd-order confined solutions have reached their asymptotic shape, as was found in Fig. 10(b).

The trajectory error of the vortex for the three grids is presented in Fig. 14 for the various cases presented above (2nd-order scheme with 1st-order confinement, 3rd-order scheme with 3rdorder confinement, 5th-order scheme without and with 1st- and 3rd-order confinement). Without confinement (i.e. with the 5thorder scheme), the vortex is rapidly dissipated on the coarser mesh, therefore the predicted vortex trajectory shows very large errors. This is no more the case with the medium and the fine 


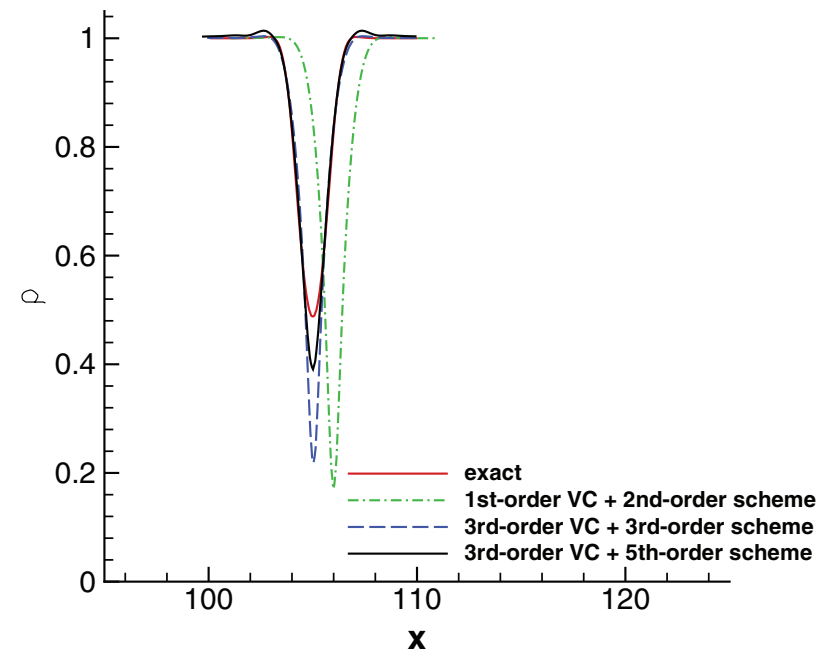

(a) fine

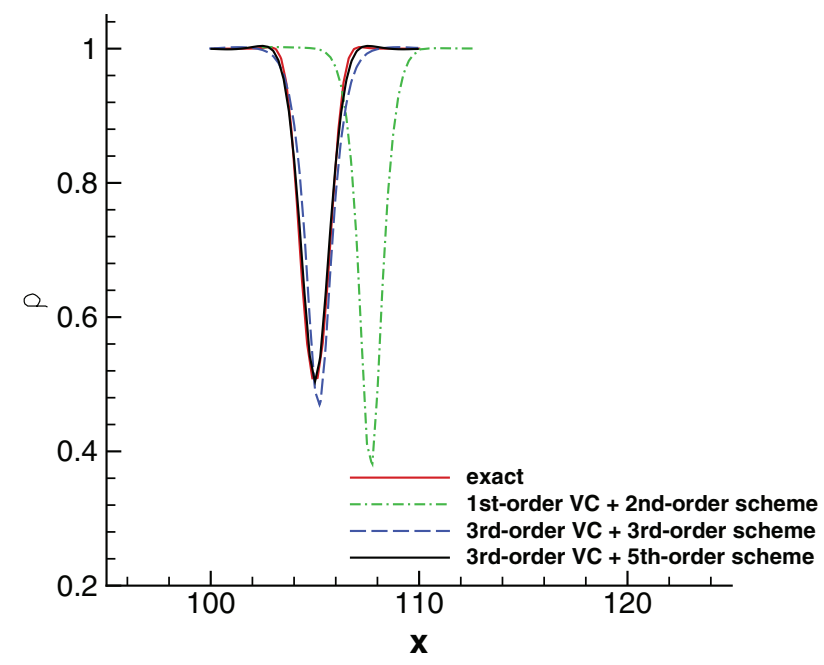

(b) medium

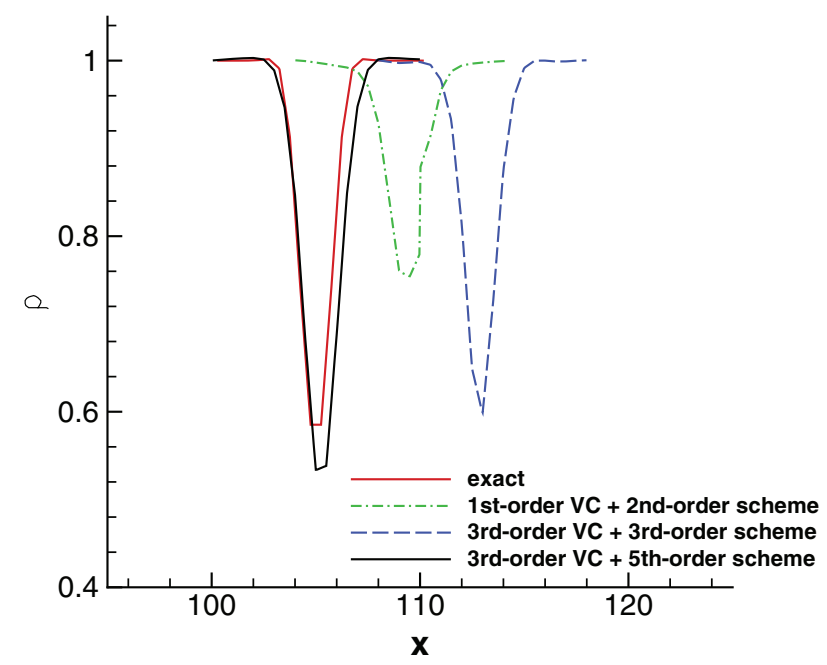

(c) coarse

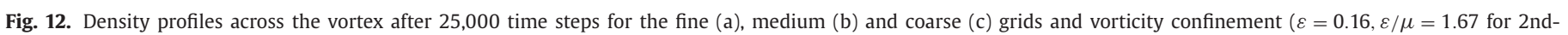
and 3rd-order schemes, $\varepsilon=0.02 / 0.04, \varepsilon / \mu=1.67$ for 5th-order scheme).

grids for which the error remains very small all along the computed period of time (the plotted errors are normalized by the vortex core radius). When confinement is applied, the error also remains small for all computations with the 5th-order scheme, generally less than one core radius. Small deviations in longitudinal vortex position are nevertheless obtained after a while with the fine grid, contrary to what is obtained without confinement. However, as is also the case for all grids, 3rd-order confinement reduces this error with respect to 1 st-order confinement. The results obtained with the 3rd-order scheme and 3rd-order confinement and with the 2nd-order scheme and 1st-order confinement are less satisfactory since significantly larger errors in vortex position are obtained. They are due to the larger dispersion error of the underlying 2nd- and 3rd-order scheme, so that the 3rd-order confinement reduces the error with respect to the 1st-order confinement. An exception is noticeable for the axial position error with the coarse mesh where the 3rd-order scheme and 3rd-order confinement produces a larger error than the 2 nd-order scheme and $1 \mathrm{st}$ order confinement. However, this is only for relatively short times of advection as the 2nd-order scheme with 1st-order confinement predicts a much larger error for larger times (see Fig. 13(c) where the vortex computed with the 2nd-order scheme has almost travelled once more across the grid). Furthermore, this larger error of the 3rd-order scheme with 3rd-order confinement is probably related to an unnecessarily large confinement parameter with respect to the truncation error of the underlying 3rd-order scheme, this parameter being set at the same value as that of the 2nd-order scheme and 1st-order confinement.

Finally, typical results of a parametric study on the sensitivity of vortex solutions to confinement parameters are shown in the following four figures. First, the effect of the parameter $\varepsilon$ is presented for a fixed value of $\frac{\varepsilon}{\mu}=1.67$. The density profiles computed with 


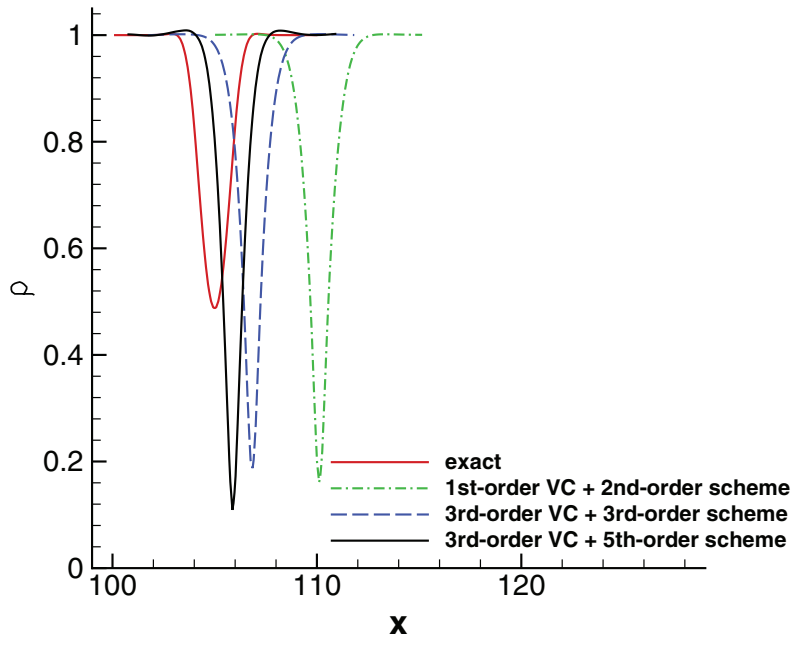

(a) fine

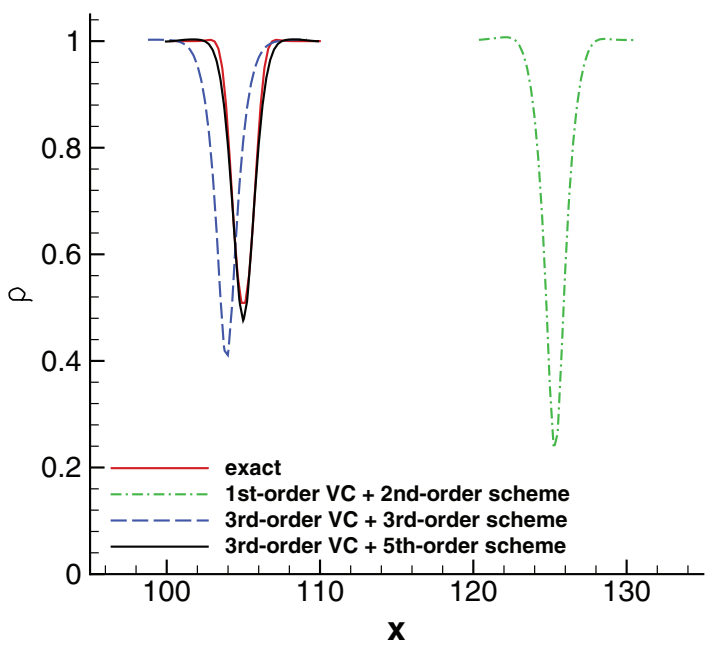

(b) medium

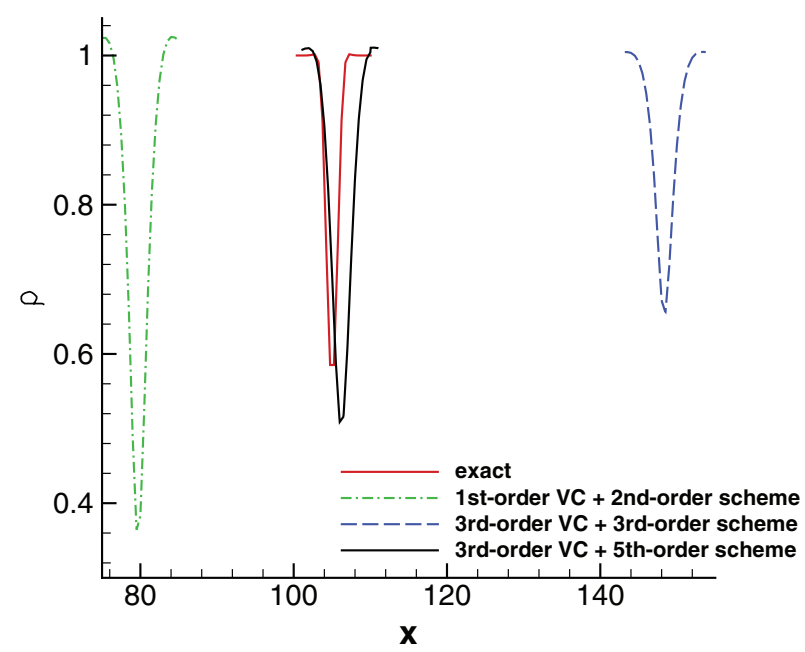

(c) coarse

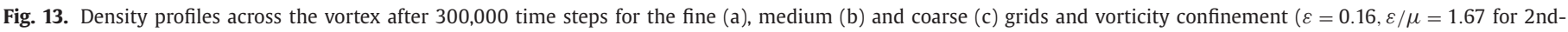
and 3rd-order schemes, $\varepsilon=0.02 / 0.04, \varepsilon / \mu=1.67$ for 5 th-order scheme).

the fine mesh are compared after 25,000 time steps in Fig. 15 with 1 st-order and 3rd-order confinement. For 1st-order confinement, the confinement parameter was varied over the range 0.00015625 $\leq \varepsilon \leq 0.005$, and with third-order confinement it was varied over the range $0.000625 \leq \varepsilon \leq 0.02$. The solutions with 3rd-order confinement are less dependent on the choice of $\varepsilon$ and closer to the exact one. After 300,000 time steps (Fig. 16), both 1st-order and 3rd-order confinement methods behave similarly. Vortex strength increases with the confinement parameter $\varepsilon$, and if the value of $\varepsilon$ is too small, the resulting vortex is weaker than the original one. In that case, the magnitude of the confinement terms is too small with respect to the artificial viscosity of the 5th-order scheme. For the larger values of $\varepsilon$ used, the vortex is slightly moved ahead of its exact position, more particularly with 3rd-order confinement, and discrepancies with respect to the exact solution begin to be significant.
For a fixed value of the confinement parameter $\varepsilon=0.01$, the effect of the diffusion/confinement ratio $\mu / \varepsilon$ is now investigated. The density profiles are shown in Fig. 17 after 25,000 time steps. The 3rd-order confinement provides results in closer agreement with the exact solution, whatever the value of $\mu / \varepsilon$, which is an effect of the higher accuracy of 3rd-order confinement. After 300,000 time steps (Fig. 18), the solutions are closer to their asymptotic state and very similar results are obtained with 1 st-order and 3rd-order confinement. The only difference actually appears for the lower values of $\mu / \varepsilon$. For $\mu / \varepsilon=0.9$, the confinement parameter $\varepsilon=0.01$ is slightly too small with 3rd-order confinement and the vortex is weaker than the exact solution.

The results of this parametric study thus show that the effect of confinement parameters is reasonably small for short to moderate times of advection (corresponding to 100 vortex radii in terms of advection distance). From this aspect, 3rd-order confinement 


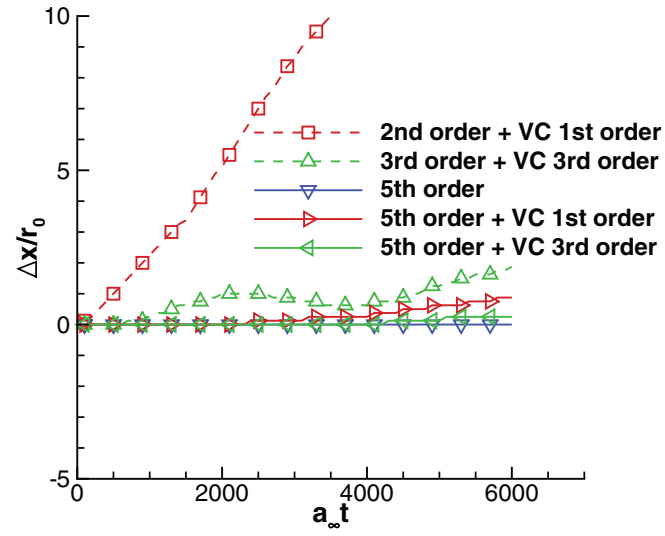

(a) $x$ trajectory error, fine

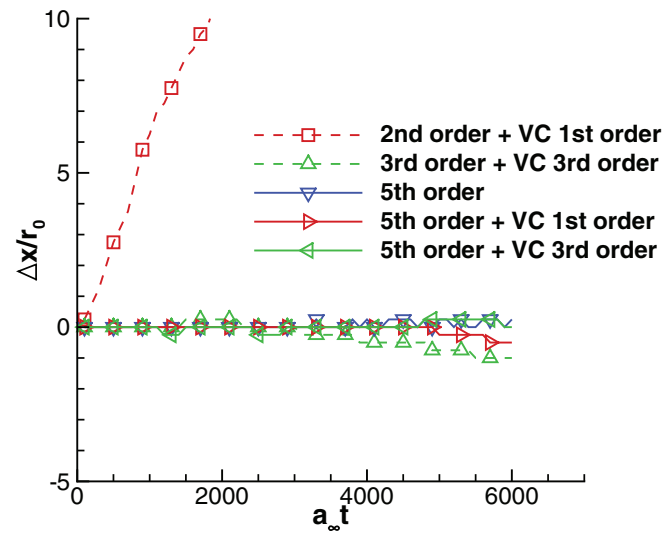

(c) $\mathrm{x}$ trajectory error, medium

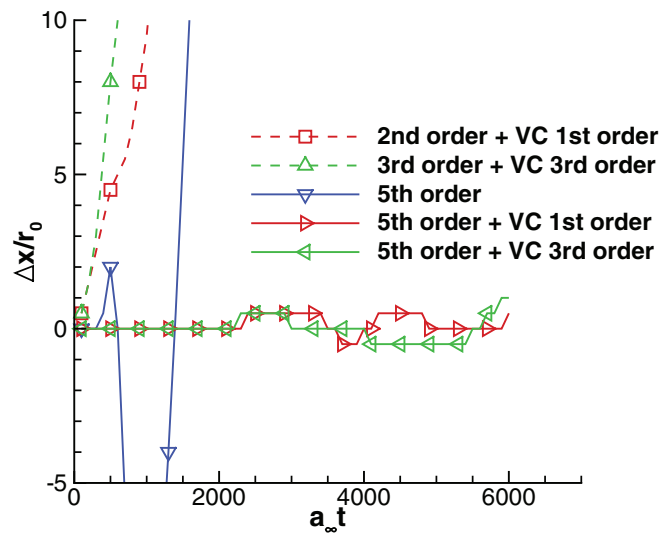

(e) $\mathrm{x}$ trajectory error, coarse

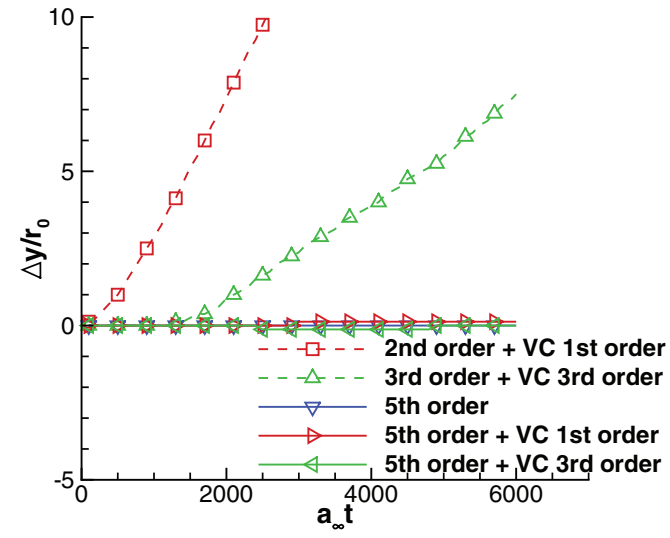

(b) y trajectory error, fine

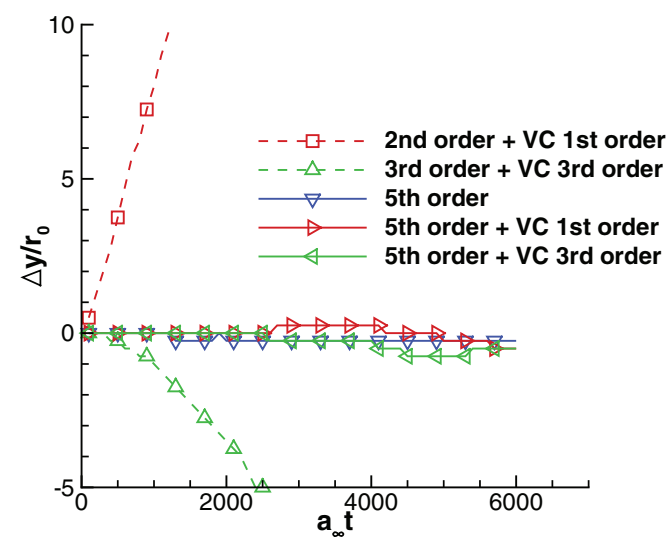

(d) y trajectory error, medium

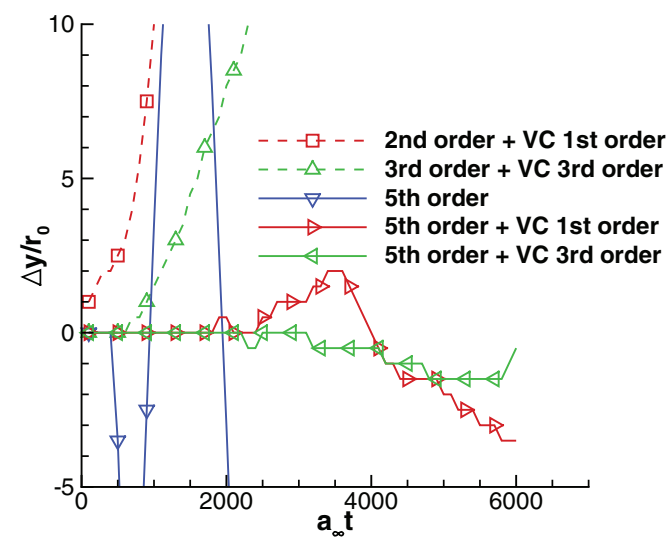

(f) y trajectory error, coarse

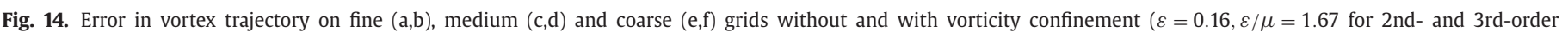
schemes, $\varepsilon=0.02 / 0.04, \varepsilon / \mu=1.67$ for 5th-order scheme). 


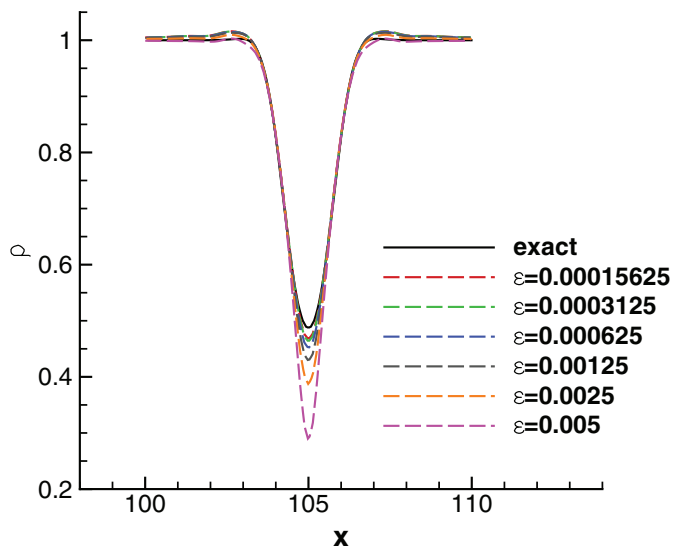

(a) 1st-order confinement

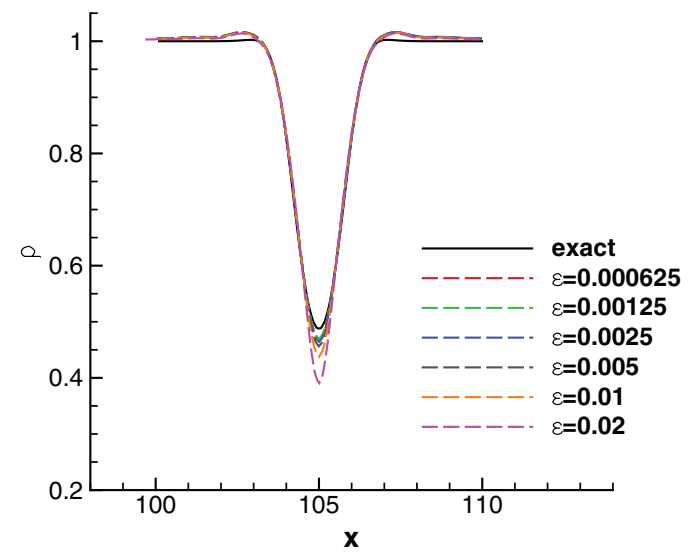

(b) 3rd-order confinement

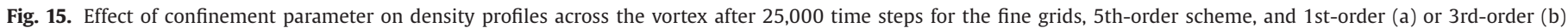
vorticity confinement $(\varepsilon / \mu=1.67)$.

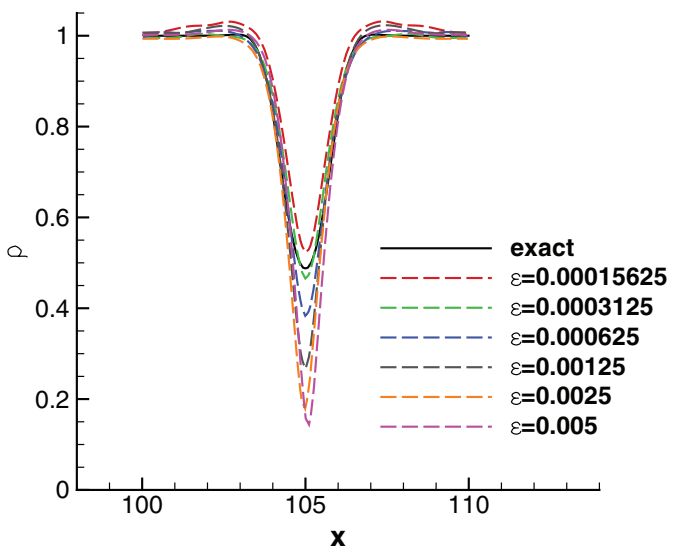

(a) 1st-order confinement

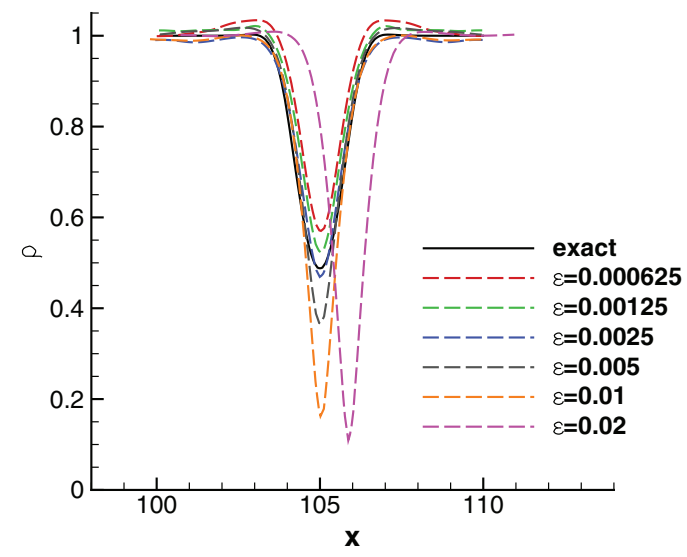

(b) 3rd-order confinement

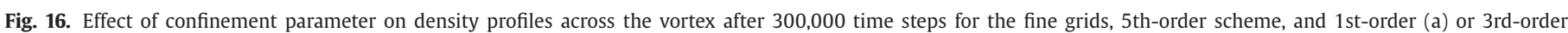
(b) vorticity confinement $(\varepsilon / \mu=1.67)$.

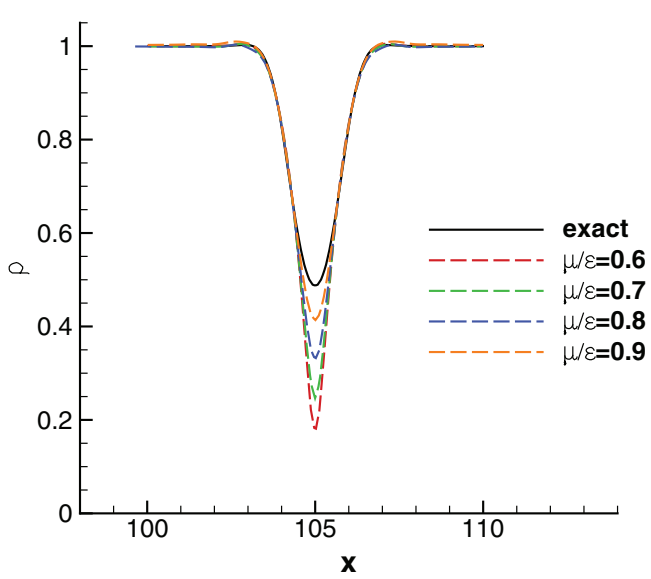

(a) 1st-order confinement

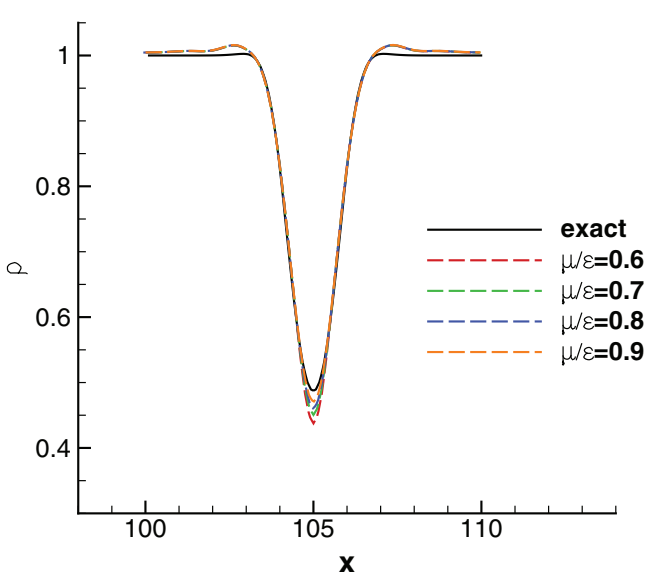

(b) 3rd-order confinement

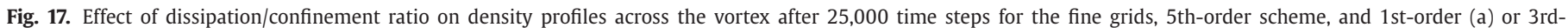
order (b) vorticity confinement $(\varepsilon=0.01)$. 


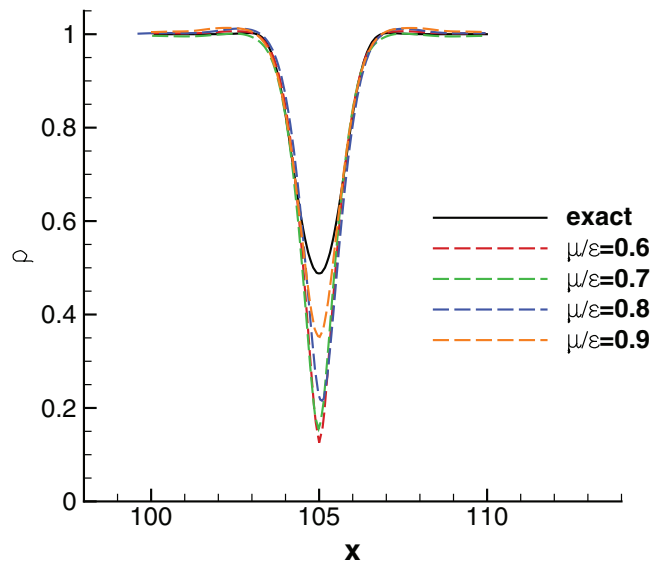

(a) 1st-order confinement

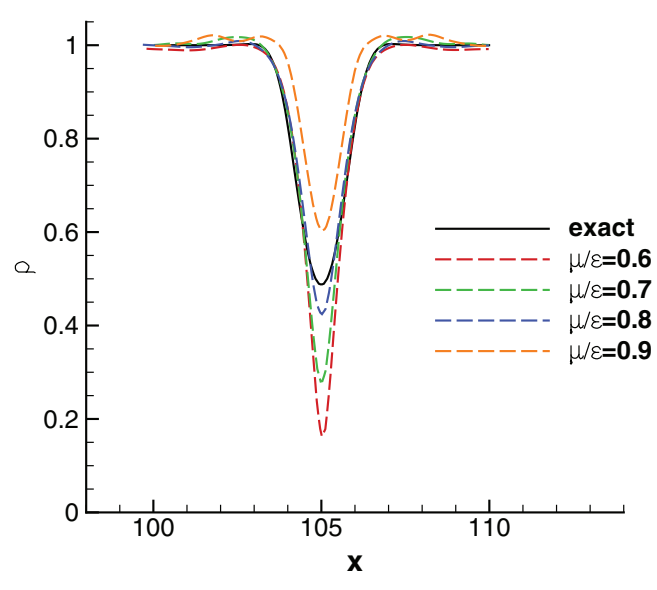

(b) 3rd-order confinement

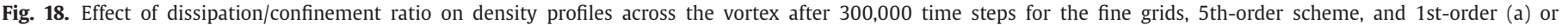
3rd-order (b) vorticity confinement $(\varepsilon=0.01)$.

is superior to 1 st-order because the asymptotic state imposed by the confinement terms is reached later and thus computed solutions are closer to the exact one. When very long periods of time are computed, both methods converge towards similar asymptotic states where the confinement parameters drive the solution. Too small values of the confinement parameter lead to a progressive dissipation of the vortex during its advection, but at a lower rate than without confinement. Too large values of the confinement parameters lead to a moderate increase of the dispersion errors. As a consequence, it is recommended to take this parameter as low as possible.

\subsection{Airfoil-vortex interaction}

\subsubsection{Test case}

The final step consists in a validation of the method in a realistic 2D unsteady BVI application. For this purpose the experiment of parallel blade-vortex interaction carried out by Kitaplioglu, Caradonna et al. in $[33,34]$ for a rotor blade equipped with a constant NACA0012 airfoil section was chosen. Here, a 2D computation of the interaction for a blade section located at $r / R=87.6 \%$ is considered. Similar configurations are commonly studied in aeroacoustics since airfoil-vortex interaction is a major source of aerodynamic noise. This case was previously studied in [24] and in the frame of the ONERA-JAXA cooperation [35]. The airfoil is set at $0^{\circ}$ incidence with a freestream Mach number $M_{\infty}=0.626$. The clockwise-rotating vortex is initialized at $x=-10 c, y=-0.25$ c using the Scully model [36]. The intensity is $\Gamma=0.2536$ and the core radius $\delta=0.162 c$.

Meshing was performed by utilizing a C-type curvilinear mesh for the NACA airfoil constructed using tools of the Cassiopée suite [37] and a set of Cartesian background meshes communicating via Chimera interpolation [38,39]. Views of the computational domain are presented in Fig. 19. The background mesh is divided into four zones of varying density. To minimize mesh size, the vortex path is not covered by a uniform Cartesian region, but the mesh is refined only close to the airfoil so that a reasonable size ratio between the NACA and background mesh is maintained (Fig. 19(b)). Studies were performed for varying background mesh density $\Delta x_{\text {min }}=0.025 / 0.0125$ on the coarse and fine grids respectively, where $\Delta x_{\min }$ refers to the thickness of the finest background region. The NACA mesh is composed of $228 \times 10$ points for the coarse mesh and $450 \times 18$ points for the fine mesh. The cell size ratio is $1 / 2$ between the two finest background zones that cover the vortex advection path and 1/3 for the other cases. The coarse and fine grids contain a total of 66.147 and 254.057 cells respectively. The approximate number of cells per vortex radius is 3 and 6 during the main part of the advection, thus corresponding to the coarse and medium mesh of the previous inviscid vortex advection study. A finer mesh of 3.252 .720 cells with $\Delta x=0.00625$ in the vortex advection region and a $914 \times 36$ point mesh for the NACA was also generated and studied as a higher-accuracy reference solution. For this case, the finest background region covers the complete vortex path during the interaction, and not only the area close to the airfoil.

\subsubsection{Solution algorithm}

Presented studies are based on the compressible Euler equations since they are representative of the problem and have been shown to produce similar results to the Navier-Stokes equations for similar applications. The same 2nd, 3rd and 5th-order centered schemes of Saunier et al. [26] as the previous studies have been used for space discretization. For each case the order of accuracy in the Cartesian and curvilinear regions is the same except for the 5th-order scheme, which is combined with a 3rd-order accurate weighted curvilinear scheme [40]. Time integration is performed using an implicit 2nd-order backward Euler algorithm and the resulting Gear equation is solved using an LU factorization. The time step is $\Delta t=0.01 / 0.005 / 0.0025$ for the coarse, fine and reference meshes respectively, so that the maximum CFL number is the same for all cases. Computations were run for non-dimensional time $t=-10$ to $t=10$ so that interaction occurs approximately at $t=0$. Finally, Chimera interpolations are 2nd-order accurate for the 2ndorder scheme and 3rd-order accurate for higher order schemes. The reference solution is computed using the 5th-order scheme. Lastly, for cases with VC, the source term is applied in Cartesian regions only and the confinement parameters were set to $\mu / \varepsilon=0.6$ and $\varepsilon=0.02$.

\subsubsection{Baseline case}

Reference results for the finer mesh with $\Delta x_{\min }=0.00625$ using the 5th-order baseline scheme are shown in Fig. 20 and show satisfactory preservation of the vortex shape during the advection phase. Profile extractions, performed horizontally for $x=0.5$ in both directions from the position of maximum absolute vorticity value, show an amplification of core vorticity by approximately $7 \%$. This might be caused by the downstream presence of the airfoil combined with the fact that the initial vortex is not an 


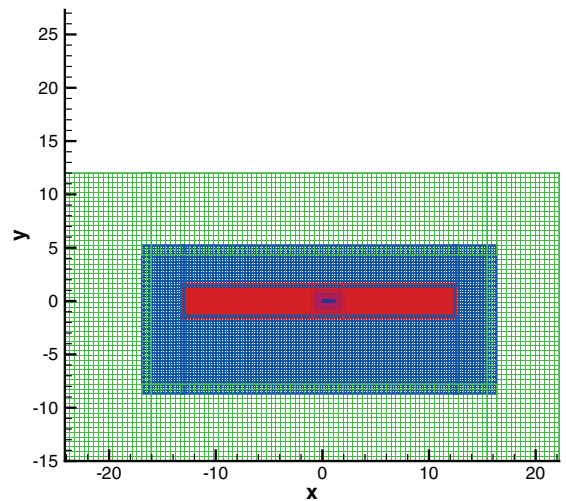

(a) Overview of the background mesh

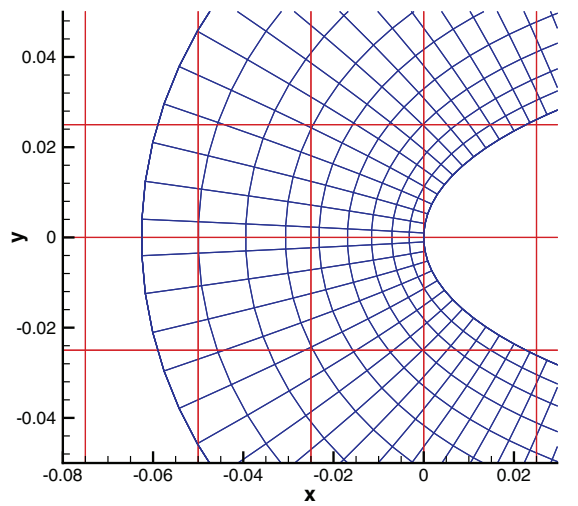

(c) Close-up view of the Leading Edge

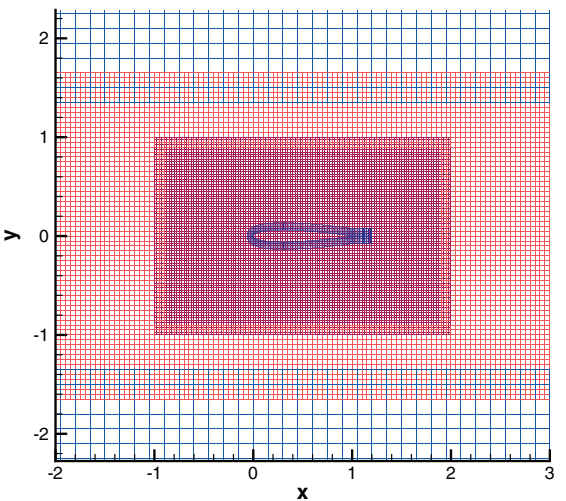

(b) NACA0012 mesh

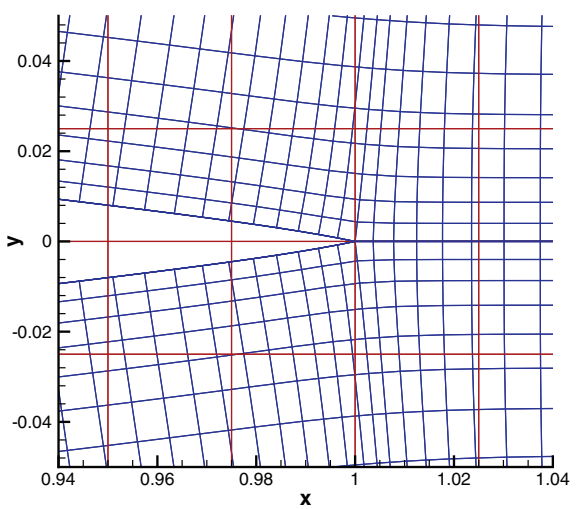

(d) Close-up view of the Trailing Edge

Fig. 19. Details of the coarse computational domain ( $\Delta x=0.025$ in the finest Cartesian region). The background mesh is divided in four zones of varying density.

exact solution of the Euler equations, meaning that it will need to deform in order to eventually relax towards an exact solution. Figs. 20(c)-20(d) display the time evolution of pressure on the airfoil surface in the upper and lower sides respectively at $x / c=0.02$. Results are compared with experimental measurements of Kitaplioglu, Caradonna et al. at the same positions. Computational results are different from the experimental measurements, probably as a result of the 3D effects of the experiment that are not accounted for in the computational study. For comparison, the computational curves are displaced so that they match the measured pressure coefficient at time $t=-7$, as in [35]. Computations produce a good approximation of the pressure evolution close to the moment of interaction, for both sides. However, a slight miscalculation of the interaction time due to phase approximation error can be observed.

Results for different scheme orders on the coarse and fine grids underline the effect of numerical error in lower-accuracy numerical schemes (Fig. 21). Diffusion of the vortex core is severe for the coarse mesh, especially for the 2nd-order computation. Isovorticity contours show that the vortex shape is noticeably distorted while additional regions of lower vorticity magnitude appear upstream of the vortex position.

Even though vorticity is better preserved as the order of the scheme increases, Table 1 shows that the pressure peaks achieved on the airfoil are less intense when the vortex is better preserved, or equivalently when a higher-accuracy scheme is used. This behaviour is related to the improved phase approximation of the scheme, rather than the reduced dissipation error, as can be seen from the vortex core position at $t=0$ as a measure of dispersion error of numerical schemes. The x-position of the vortex is related to the interaction time and the $\mathrm{y}$-position is related to the interac-
Table 1

Vortex position at $t=0$ and interaction pressure peaks at $x / c=0.02$ for the standard case.

\begin{tabular}{llllll}
\hline Case & Scheme order & $x_{\text {core }}$ & $y_{\text {core }}$ & $\max \left(C_{p U}\right)$ & $\min \left(C_{p L}\right)$ \\
\hline Experiment & - & - & - & 0.3939 & -0.4715 \\
Reference & 5th & -0.2094 & -0.2781 & 0.3921 & -0.4583 \\
Coarse mesh & 2nd & -0.2125 & -0.0375 & 0.4565 & -0.5908 \\
& 3rd & -0.0875 & -0.1875 & 0.4445 & -0.5530 \\
\multirow{2}{*}{ Fine mesh } & 5th & -0.0875 & -0.1875 & 0.4334 & -0.5468 \\
& 2nd & -0.0563 & -0.1938 & 0.4413 & -0.5194 \\
& 3rd & -0.0688 & -0.2688 & 0.4114 & -0.4895 \\
& 5th & -0.0813 & -0.2688 & 0.4098 & -0.4869 \\
\hline
\end{tabular}

tion intensity. The small vertical displacement of the vortex for the reference computation with regard to the initialization can be attributed either to the airfoil presence in the flow field or to the dispersion error of the 5th-order scheme. For the 2nd-order scheme, the vortex is positioned closer to the airfoil in the y-direction than for the other cases, both for the coarse and fine mesh. Especially for the 2nd-order scheme on the coarse mesh, increased dispersion error leads to significant amplification of the interaction. The effect of this error can be observed as an increase of the interaction pressure peak on both sides. For higher orders the dispersion error is lower, so the interaction pressure peaks are reduced even if vortex intensity is better-preserved.

The computed vortex of the lower-accuracy 2nd-order computation on the coarse mesh is positioned further from the airfoil in the $\mathrm{x}$-direction, which is fortuitously in agreement with the reference computation. This error is consistent with the miscalculation of the time of the interaction pressure peak 


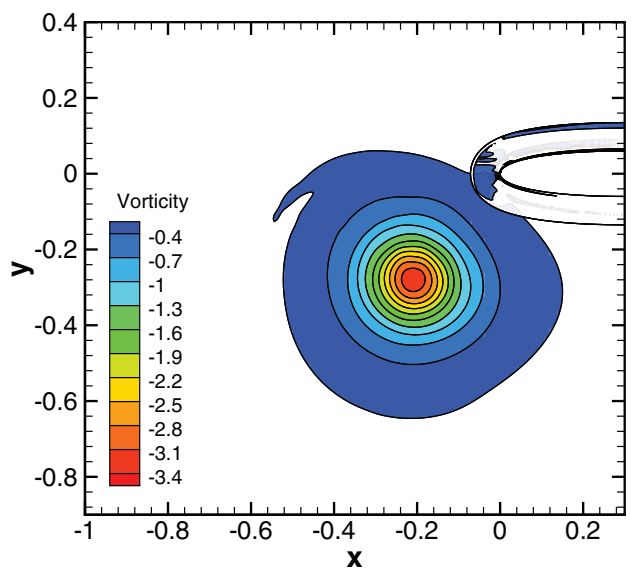

(a) Iso-vorticity contour at $t=0$

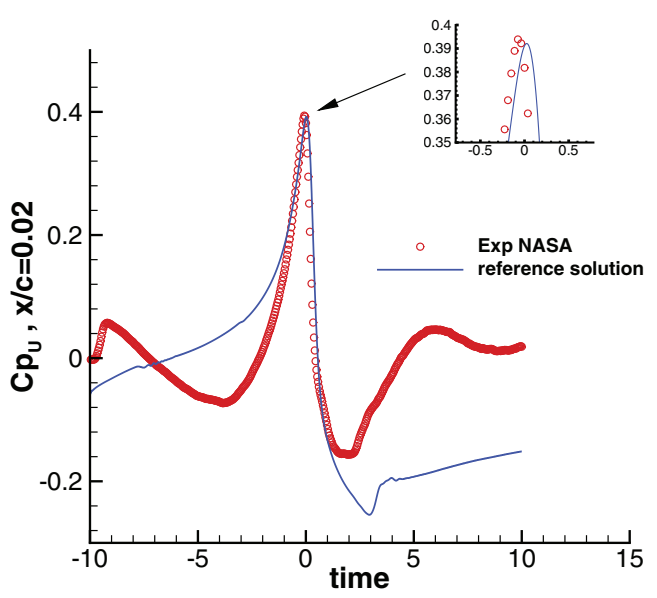

(c) Time evolution of pressure coefficient at $x / c=0.02$, upper side

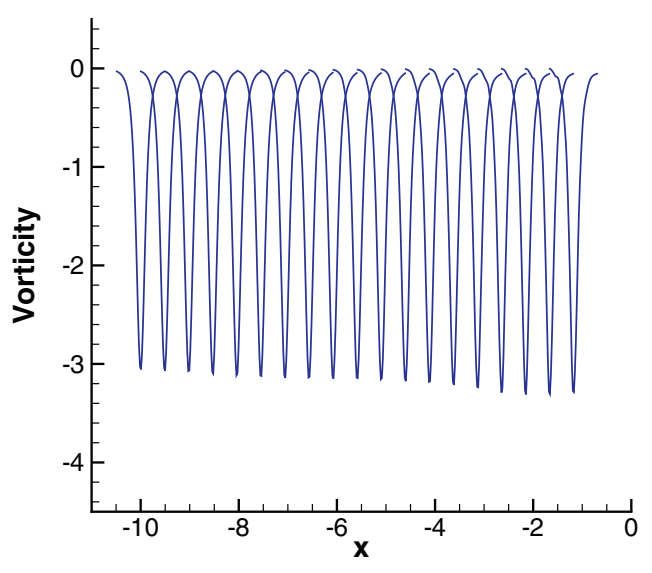

(b) Vorticity profile during advection

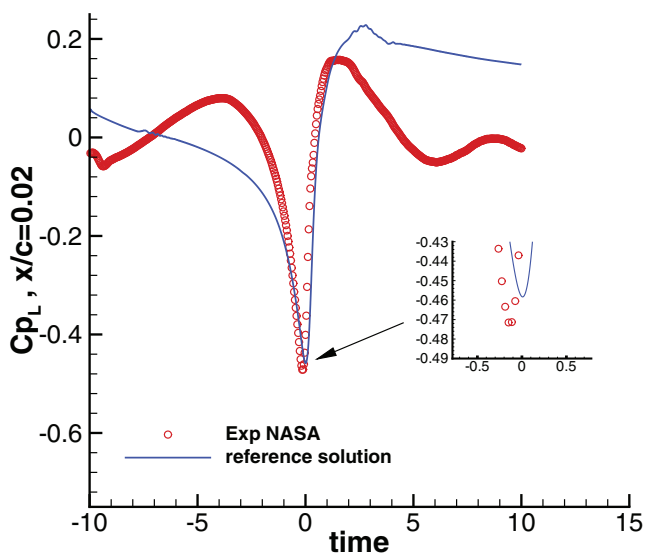

(d) Time evolution of pressure coefficient at

$x / c=0.02$, lower side

Fig. 20. Reference solution with $\Delta x=0.00625$ during the complete advection distance, 5th-order scheme.

(Figs. 20(c)-20(d)). All other cases show a different vortex position, meaning that for the reference solution this error might be connected to the relaxation of the vortex shape towards an exact solution of the Euler equations, an effect that is observed when the simulation precision is high enough. For the 2nd-order computation on the coarse mesh, the miscalculation is a result of dispersion error of the lower-accuracy scheme.

\subsubsection{Effect of confinement}

The original 1st-order confinement term acts rapidly on vorticity regions and dominates the solution thus compromising the higher precision of the underlying 5th-order scheme (Fig. 22). This overly confining property of the original method is more apparent on the fine mesh, where the vortex is already better preserved, while 3rd-order confinement presents increased compatibility with the higher-order underlying scheme. Indeed, iso-vorticity contours and profile extractions show that the higher-order confinement formulation confines vortical regions at a rate that better matches the precision of the high-order underlying scheme, thus allowing to preserve vorticity without altering the structure of the vortex (Figs. 22-23).
For the case of a 2D isolated vortex it was found that confinement does not introduce any modification to the dispersive parts of the flow equations. The simplifcations made in the vorticity transport equation (leading to a simple advection equation for a $2 \mathrm{D}$ isolated vortex) do not necessarily apply for more complex applications, but the vortex position at $t=0$, presented in Table 2, shows that confinement introduces small or no modification in vortex trajectory. Specifically, a difference is observed only in the x-direction on the coarse mesh and is the same for both orders of confinement while the y-position, which determines the strength of the interaction, is not modified. On the fine mesh, where the case is better-resolved, confinement does not modify the vortex position at the moment of interaction.

The values of pressure peaks on the upper and lower side of the airfoil are presented in the same table. As expected, absolute values of the interaction pressure increase when confinement is applied, or equivalently when the vortex intensity is stronger. It is important to note that the interest of this case is to show that confinement is compliant with the physics of the airfoil-vortex interaction rather than significantly improve the calculation of the peak pressure, since the computed pressure itself is not directly 

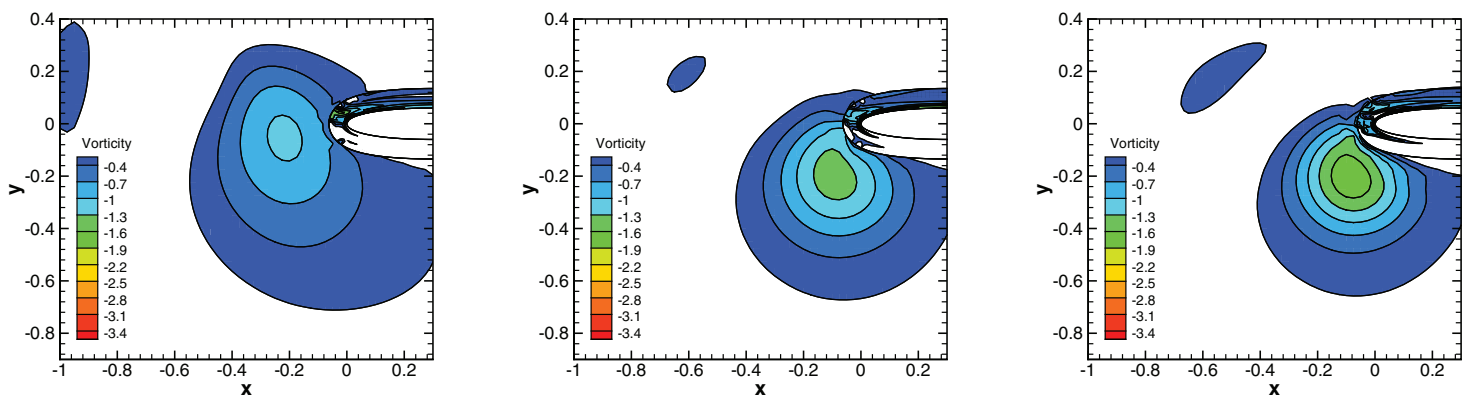

(a) coarse mesh
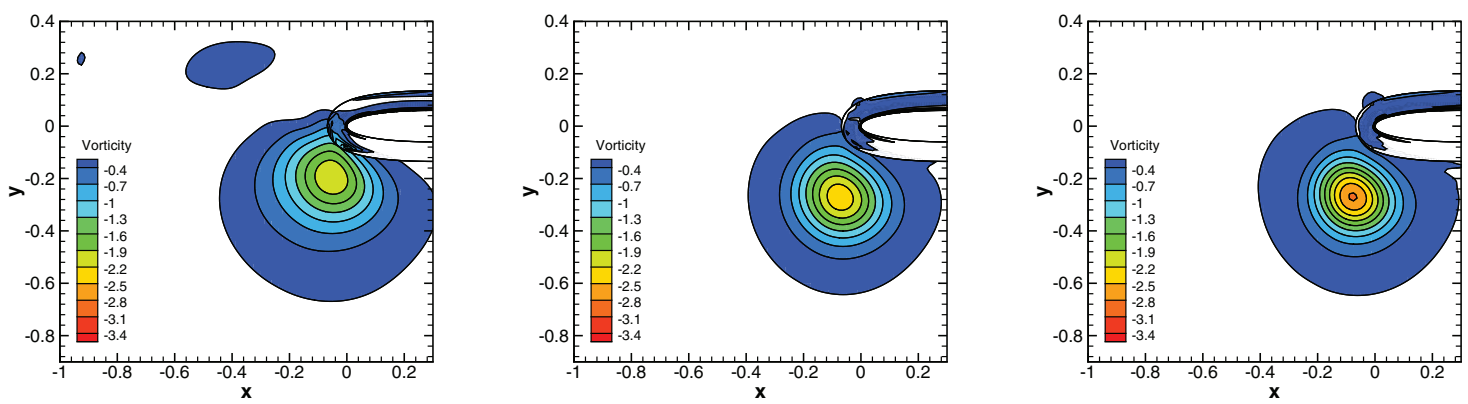

(b) fine mesh

Fig. 21. Iso-vorticity contours at $t=0$ for 2 nd-order (left), 3rd-order (middle) and 5 th-order schemes (right).
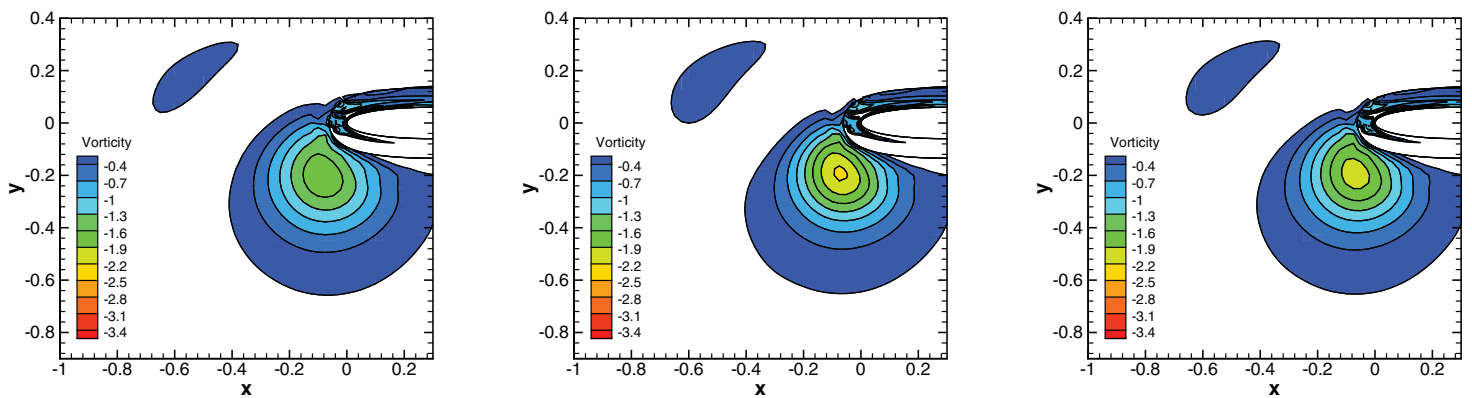

(a) coarse mesh
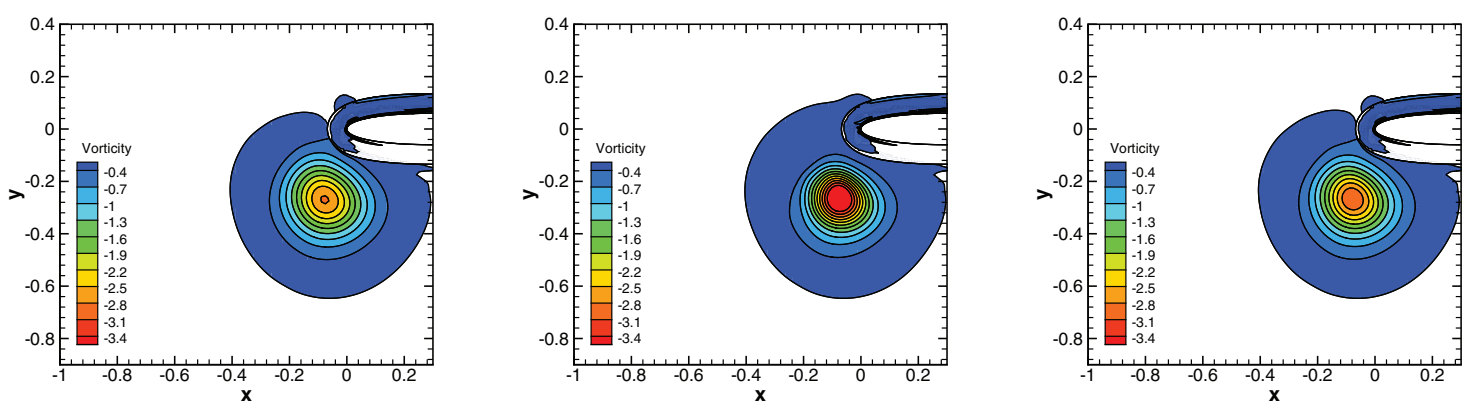

(b) fine mesh

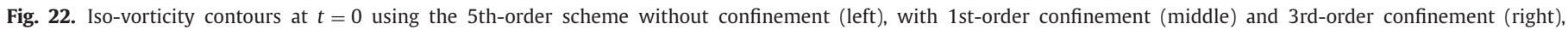
$\mu / \varepsilon=0.6$ and $\varepsilon=0.02$.

comparable to the 3D experiment as mentioned above. Similarly to the baseline schemes without confinement, the absolute peak values of computed pressure are increased compared to the reference case due to the dispersion error of the underlying 5thorder scheme, especially in the y-direction, which results in a stronger interaction. Finally, Fig. 24 shows that confinement does not disturb the stabilization of the flow after $t=0$, further proving the compliance of the method with the interaction physics. Even though the use of a VC term of the same order of accuracy as the baseline scheme would be preferable, this application 


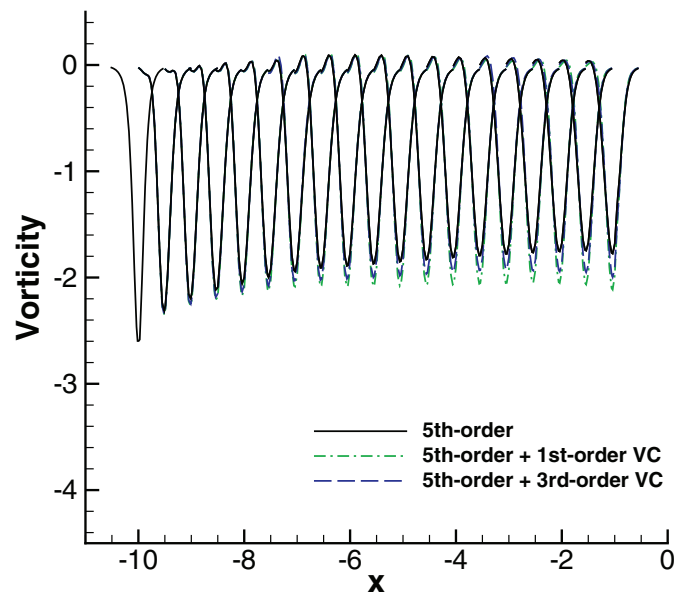

(a) coarse mesh

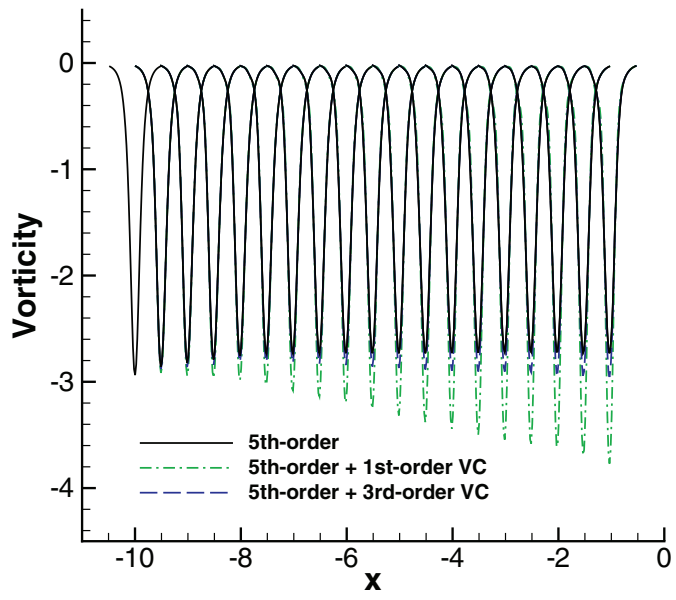

(b) fine mesh

Fig. 23. Vorticity profile over time using the 5th-order scheme and vorticity confinement, $\mu / \varepsilon=0.6$ and $\varepsilon=0.02$.

Table 2

Vortex position at $t=0$ and interaction pressure peaks at $x / c=0.02$ with vorticity confinement.

\begin{tabular}{lllllll}
\hline Case & Scheme order & VC order & $x_{\text {core }}$ & $y_{\text {core }}$ & $\max \left(C_{p U}\right)$ & $\min \left(C_{p L}\right)$ \\
\hline Experiment & - & - & - & - & 0.3939 & -0.4715 \\
Reference & 5 th & - & -0.2094 & -0.2781 & 0.3921 & -0.4583 \\
Coarse mesh & 5 th & - & -0.0875 & -0.1875 & 0.4334 & -0.5468 \\
& $"$ & 1 st & -0.0625 & -0.1875 & 0.4360 & -0.5487 \\
& $"$ & $3 r d$ & -0.0625 & -0.1875 & 0.4346 & -0.5479 \\
Fine mesh & 5th & - & -0.0813 & -0.2688 & 0.4098 & -0.4869 \\
& $"$ & 1 st & -0.0813 & -0.2688 & 0.4113 & -0.4884 \\
& $"$ & 3rd & -0.0813 & -0.2688 & 0.4105 & -0.4877 \\
\hline
\end{tabular}

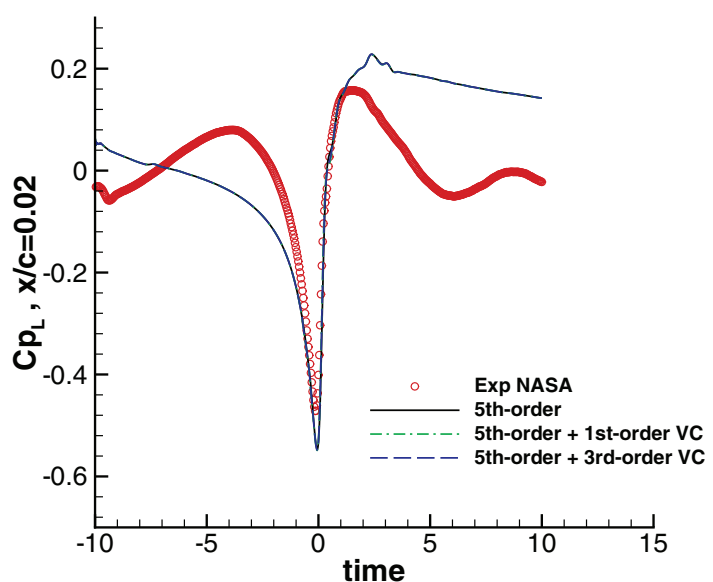

(a) coarse mesh

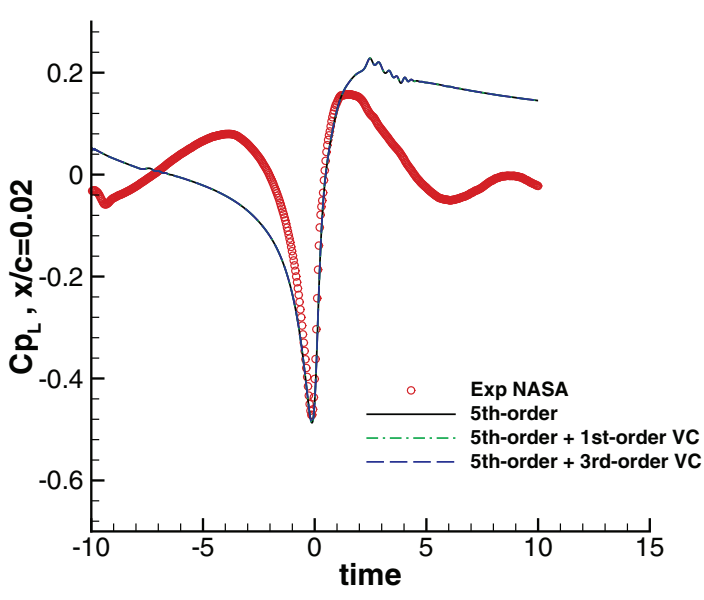

(b) fine mesh

Fig. 24. Time evolution of pressure coefficient on the lower side at $x / c=0.02$ using the 5th-order scheme and vorticity confinement, $\mu / \varepsilon=0.6$ and $\varepsilon=0.02$.

demonstrates that the third-order VC scheme can achieve improved vorticity preservation without deteriorating the solution due to the introduction of lower-order terms in the flow equations.

\section{Conclusions}

A third-order accurate vorticity confinement scheme has been developed for the Euler and RANS equations on Cartesian grids. It is a generalization of previously developed high-order confinement schemes for the scalar advection equation. In this last case, the- ory and numerical experience show that computed structures relax asymptotically to an analytical shape that depends on the computational grid. Furthermore, higher-order confinement schemes relax to the asymptotic solution in a slower rate than lower-order ones, a property that is in line with their reduced numerical error.

In the case of the Euler/RANS equations, the procedure is simply based on the Laplacian of the original VC2 term and is conservative. The analogy of the VC formulation with the linear advection case was shown by an analysis of the vorticity transport equation, resulting to a similar form for the asymptotic solution. A 
grid convergence study demonstrates the actual order of accuracy of the developed scheme and shows that the application of VC does not have a negative effect on any particular flow variable such as entropy.

Further numerical tests prove that the new scheme combines the confinement capability of the original VC2 scheme with the increased precision of the higher-order. An advection study of a 2D vortex over very long distances shows that vortices eventually diffuse, even for high-order schemes, but VC allows to preserve vorticity without altering the vortex structures. Additionally, the thirdorder VC scheme was shown to be more precise in terms of predicted vortex intensity and trajectory than its first-order counterpart, both for short and long advection distances. Finally, an application of the method in an airfoil-vortex interaction case shows that third-order VC presents increased compatibility with higherorder schemes and produces consistent results with the previous studies without modifying the interaction physics.

The developed method allows for preservation of vortical structures over long distances with little cost compared to finer mesh computations and without special treatment for different applications. Due to its increased precision, the third-order VC scheme acts in a rate that better matches the numerical error of high-order schemes. As such, it can be a useful tool for problems dominated by dissipation, even if it can introduce small dispersion errors. Furthermore, the derivation procedure of 3rd-order VC can be recursively performed to obtain even higher orders for use with higherorder baseline schemes.

\section{Acknowledgements}

Part of this research was supported by a DGA-MRIS scholarship.

\section{References}

[1] Winckelmans G, Cocle R, Dufresne L, Capart R. Vortex methods and their application to trailing wake vortex simulations. Comptes Rendus Physique 2005;6(45):467-86. http://dx.doi.org/10.1016/j.crhy.2005.05.001.Aircraft trailing vortices; http://www.sciencedirect.com/science/article/pii/ S1631070505000575.

[2] Leishman JG, Bhagwat MJ, Bagai A. Free-vortex filament methods for the analysis of helicopter rotor wakes. J Aircraft 2002;39(5):759-75.

[3] Joubarne E, Guibault F, Braun O, Avellan F. Numerical capture of wing tip vortex improved by mesh adaptation. Int J Numer Methods Fluids 2011;67(1):8-32.

[4] Shenoy R, Smith MJ, Park MA. Unstructured overset mesh adaptation with turbulence modeling for unsteady aerodynamic interactions. J Aircraft 2014;51(1):161-74.

[5] Hariharan N, Steffen M, Wissink A, Potsdam M. Tip vortex field resolution using an adaptive dual-mesh computational paradigm. in: 49th AIAA aerospace sciences meeting including the new horizons forum and aerospace exposition, AIAA 2011, 1108; 2011.

[6] Chaderjian NM, Ahmad JU. Detached eddy simulation of the uh-60 rotor wake using adaptive mesh refinement. In: Proceedings of the 68th annual forum of the American helicopter society, Forth Worth, TX; 2012.

[7] Kamkar S, Wissink AM, Sankaran V, Jameson A. Feature-driven cartesian adaptive mesh refinement for vortex-dominated flows. J Comput Phys 2011;230(16):6271-98.

[8] Kamkar SJ. Mesh adaption strategies for vortex-dominated flows. Stanford University; 2011. Ph.D. thesis.

[9] Frey P-J, Alauzet F. Anisotropic mesh adaptation for cfd computations. Comput Methods Appl Mech Eng 2005;194(48):5068-82.

[10] Fidkowski KJ, Roe PL. An entropy adjoint approach to mesh refinement. SIAM J Sci Comput 2010;32(3):1261-87.

[11] Shu C-W. High-order finite difference and finite volume weno schemes and discontinuous Galerkin methods for cfd. Int J Comput Fluid Dyn 2003; 17(2):107-18.

[12] Chaderjian NM. Advances in rotor performance and turbulent wake simulation using des and adaptive mesh refinement. in: 7th international conference on computational fluid dynamics, Big Island, Hawaii; 2012.

[13] Grimich K, Michel B, Cinnella P, Lerat A. An accurate finite-volume formulation of a Residual-Based Compact scheme for unsteady compressible flows. Comput Fluids 2014;92:93-112. http://dx.doi.org/10.1016/j.compfluid.2013.12.016. http: //www.sciencedirect.com/science/article/pii/S0045793013004969.

[14] Steinhoff J, Lynn N. Treatment of vortical flows using vorticity confinement. Chapter 10 of computing the future IV: frontiers of computational fluid dynamics. Springer-Verlag; 2006.
[15] Steinhoff J, Lynn N, Yonghu W, Fan M, Wang L, Dietz W. Turbulent flow simulations using vorticity confinement. Chapter 12 of implicit large eddy simulations: computing turbulent flow dynamics. Cambridge University Press; 2006.

[16] Steinhoff J, Lynn N, Wang L. Large eddy simulation using vorticity confinement. Chapter 4 of implicit large eddy simulations: computing turbulent flow dynamics. Cambridge University Press; 2006.

[17] Costes M, Kowani G. An automatic anti-diffusion method for vortical flows based on vorticity confinement. Aerosp Sci Technol 2003;7(1):11-21. http://dx.doi.org/10.1016/S1270-9638(02)01180-X. http://www.sciencedirect. com/science/article/pii/S127096380201180X.

[18] Costes M. Analysis of the second vorticity confinement scheme. Aerosp Sci Technol 2008;12(3):203-13. http://dx.doi.org/10.1016/j.ast.2007.06.004. http:// www.sciencedirect.com/science/article/pii/S1270963807000831.

[19] Costes M, Renaud T, Rodriguez B, Reboul G. Application of vorticity confinement to rotor wake simulations. Int J Eng Syst Modell Simul 2012;4(1/2):10212. doi:10.1504/IJESMS.2012.044848.

[20] Costes M. Stability analysis of the $\{\mathrm{VC} 2\}$ confinement scheme for the linear transport equation. Comput Fluids 2013;86(0):537-57. http://dx.doi.org/10. 1016/j.compfluid.2013.07.026. http://www.sciencedirect.com/science/article/pii/ S0045793013003058.

[21] Steinhoff J, Puskas E, Babu S, Wenren Y, Underhill D. Computation of thin features over long distances using solitary waves. AIAA paper 97-1976, 13th computational fluid dynamics conference. 13th computational fluid dynamics conference; Snowmass, Colorado; 1997.

[22] Steinhoff J, Dietz W, Haas S, Xiao M, Lynn N, Fan M. Simulating small scale features in fluid dynamics and acoustics as nonlinear solitary waves. AIAA paper 2003-078, 41st aerospace meeting and exhibit. Reno, Nevada; 2003.

[23] Costes M, Juillet F. Analysis and higher-order extension of the VC2 confinement scheme. Comput Fluids 2012;56(0):102-17. http://dx.doi.org/10. 1016/j.compfluid.2011.12.002. http://www.sciencedirect.com/science/article/pii/ S0045793011003653.

[24] Costes M. Development of a 3rd-order vorticity confinement scheme for rotor wakes simulations. In: 38th European rotorcraft forum. Amsterdam, The Netherlands; 2012

[25] Saunier O. Méthode d'adaptation de maillages cartésiens basée sur des schémas d'ordre élevé pour les équations d'euler d'un fluide compressible. application aux pales de rotor d'hélicoptére. ENSAM Paris; 2008. Ph.D. thesis.

[26] Saunier O, Peron S, Jeanfaivre G, Benoit C, Lerat A. High-order accurate cartesian partitioning methods. application to rotor flows. 33rd European rotorcraft forum, Kazan, Russia. 33rd european rotorcraft forum; Kazan, Russia; 2007.

[27] Lerat A, Corre C. High-order residual-based compact schemes on structured grids. von Karman Lecture Series 2006-01. von Karman Institute for Fluid Dynamics; 2005.

[28] Steger JL, Benek JA. On the use of composite grid schemes in computational aerodynamics. Comput Methods Appl Mech Eng 1987;64(13):301-20. http://dx.doi.org/10.1016/0045-7825(87)90045-4. http://www.sciencedirect. com/science/article/pii/0045782587900454.

[29] Jeanfaivre G, Benoit C, Pape MCL. Improvement of the robustness of the chimera method. In: Fluid dynamics and co-located conferences. American Institute of Aeronautics and Astronautics; 2002. doi:10.2514/6.2002-3290.

[30] Yee H, Sandham N, Djomehri M. Low-dissipative high-order shock-capturing methods using characteristic-based filters. J Comput Phys 1999;150(1):199238. http://dx.doi.org/10.1006/jcph.1998.6177. http://www.sciencedirect.com/ science/article/pii/S0021999198961770.

[31] Yee H, Vinokur M, Djomehri M. Entropy splitting and numerical dissipation. J Comput Phys 2000;162(1):33-81. http://dx.doi.org/10.1006/jcph.2000.6517. http://www.sciencedirect.com/science/article/pii/S0021999100965173.

[32] Visbal MR, Gaitonde DV. On the use of higher-order finite-difference schemes on curvilinear and deforming meshes. J Comput Phys 2002;181(1):15585. http://dx.doi.org/10.1006/jcph.2002.7117. http://www.sciencedirect.com/ science/article/pii/S0021999102971172.

[33] Kitaplioglu C, Caradonna F. Aerodynamics and acoustics of blade-vortex interaction using an independently generated vortex. In: AHS specialists conference. San Francisco, California; 1994.

[34] Kitaplioglu C, Caradonna F, Burley C. Parallel blade-vortex interactions: an experimental study and comparison with computations. J Am Helicopter Soc 1997;42(3):272-81.

[35] Peron S, Benoit C, Renaud T, Sidès J, Tanabe Y, Saito S, et al. ONERA/JAXA common investigations on CFD tools for an accurate prediction of BVI In: AHS specialists meeting, Seoul, Korea. AHS specialists meeting; Seoul, Korea; 2007.

[36] Scully MP. Computation of helicopter rotor wake geometry and its influence on rotor harmonic airloads. Massachusetts Institute of Technology; 1975. Ph.D. thesis. http://hdl.handle.net/1721.1/64826.

[37] Benoit C, Péron S, Landier S. Cassiopee: A \{CFD $\}$ pre- and post-processing tool. Aerosp Sci Technol 2015;45:272-83. http://dx.doi.org/10.1016/j.ast.2015.05.023. http://www.sciencedirect.com/science/article/pii/S1270963815001777.

[38] Steger JL, Dougherty FC, Benek JA. A chimera grid scheme. Adv Grid Gen 1983;5(2):5.

[39] Benek J, Steger J, Dougherty FC. A flexible grid embedding technique with application to the Euler equations. Fluid dynamics and co-located conferences. American Institute of Aeronautics and Astronautics; 1983. doi:10.2514/ 6.1983-1944.

[40] Rezgui A, Cinnella P, Lerat A. Third-order accurate finite volume schemes for euler computations on curvilinear meshes. Comput Fluids 2001;30(78):875-901. http://dx.doi.org/10.1016/S0045-7930(01)00033-0. http://www.sciencedirect.com/science/article/pii/S0045793001000330. 